\title{
Collection strategy, inner morphology, and size distribution of dust particles in ASDEX Upgrade
}

\author{
M Balden ${ }^{1,4}$, N Endstrasser ${ }^{1}$, P W Humrickhouse ${ }^{2}$, V Rohde ${ }^{1}$, M Rasinski ${ }^{1,3}$, \\ $\mathrm{U}_{\text {von Toussaint }}{ }^{1}, \mathrm{~S}$ Elgeti $^{1}$, $\mathrm{R} \mathrm{Neu}^{1}$ and the ASDEX Upgrade Team ${ }^{1}$ \\ ${ }^{1}$ Max-Planck-Insitut für Plasmaphysik, Euratom Association, \\ Boltzmannstrasse 2, D-85748 Garching, Germany \\ ${ }^{2}$ Idaho National Laboratory, P.O. Box 1625, Idaho Falls, Idaho 83415, USA \\ ${ }^{3}$ Warsaw University of Technology, Faculty of Material Science and \\ Engineering, Woloska 141, 02-507, Poland
}

E-mail: Martin.Balden@ipp.mpg.de

\begin{abstract}
The dust collection and analysis strategy in ASDEX Upgrade (AUG) is described. During five consecutive operation campaigns (2007-2011), Si collectors were installed, which were supported by filtered vacuum sampling and collection with adhesive tapes in 2009. The outer and inner morphology (e.g. shape) and elemental composition of the collected particles were analysed by scanning electron microscopy. The majority of the $\sim 50000$ analysed particles on the Si collectors of campaign 2009 contain tungsten - the plasma-facing material in AUG - and show basically two different types of outer appearance: spheroids and irregularly shaped particles. By far most of the $\mathrm{W}$-dominated spheroids consist of a solid $\mathrm{W}$ core, i.e., solidified W droplets. A part of these particles is coated with low Z material; a process which seems to happen presumably in the far scrape off layer plasma. In addition, some conglomerates of $\mathrm{B}, \mathrm{C}$, and $\mathrm{W}$ appear as spherical particles after their contact with plasma. By far most of the particles classified as B-, C-, and W-dominated irregularly shaped particles consist of the same conglomerate with varying fraction of embedded $\mathrm{W}$ in the B-C matrix and some porosity, which can exceed $50 \%$. The fragile structures of many conglomerates confirm the absence of intensive plasma contact. Both the ablation and mobilization of conglomerate material and the production of $\mathrm{W}$ droplets are proposed to be triggered by arcing. The size distribution of each dust particle class is best described by a lognormal distribution allowing an extrapolation of the dust volume and surface area. The maximum in this distribution is observed above the resolution limit of $0.28 \mu \mathrm{m}$ only for the $\mathrm{W}$-dominated spheroids, at around $1 \mu \mathrm{m}$. The amount of $\mathrm{W}$-containing dust is extrapolated to be less than $300 \mathrm{mg}$ on the horizontal areas of AUG.
\end{abstract}

PACS: 52.27.Lw, 52.40.Hf , 28.52.Nh, 68.37.Hk, 82.80.Ej

Keywords: dust, particles size distribution, tungsten, tokamak, SEM

Submitted to: Nuclear Fusion

${ }^{4}$ Author to whom any correspondence should be addressed. 


\section{Introduction}

Accidental air or steam ingress during operation of ITER and further future device can lead to significant production of explosive hydrogen on the reactive surfaces of dust particles accumulated in the device. Furthermore, dust mobilization is possibly followed by a dust explosion (oxidation) and long-range transport of radioactive nuclides embedded therein. These scenarios are vastly influenced by the morphological properties of the dust particles, such as particle size, specific surface area and elemental composition $\left[^{[-5}\right]$. Therefore, it is of high importance to know which different types of dust exist in fusion devices. Beyond the radiological safety aspect of dust, its effects on the plasma performance and operation have to be considered $\left[2,{ }^{6}\right]$. Therefore, the dust generation mechanisms, its mobilization, transport and deposition pattern have to be investigated and quantified.

Several dust generation processes are suggested $\left[2,{ }^{7}\right]$ and the research of dust in fusion devices was reviewed recently $\left[3,{ }^{8-12}\right]$ including in-situ observation with modelling and ex-situ post-mortem analyses. The remark must be made that in-situ observation of dust events, especially of light emitting particles with fast cameras, can hardly be correlated to the populations of the different dust types and size distributions observed by post-mortem analyses. Furthermore, re-distribution of the dust particles inside the devices has to be considered (e.g., during vents and mechanical vibrations due to disruptions) for the comparison between dust transport modelling and observed post-mortem distributions.

Beside its impact on the development of operation scenarios and safety measures for future fusion power plants, research on dust particles in a plasma environment (dusty plasma) is also of importance, e.g., for industrial processing, especially in the semiconductor industry, atmospheric studies, geophysics, and astrophysics $\left[{ }^{13-16}\right]$. From dusty plasma physics, knowledge is adapted to fusion $\left.{ }^{17,18}\right]$.

Since the late 1990s particles have been collected in various fusion plasma devices during vents for post-mortem analysis $\left[2,18,{ }^{19-35}\right]$, and several attempts were made to model the behaviour of dust particles in a fusion plasma $\left[3,11,12,{ }^{36-42}\right]$. In ASDEX Upgrade (AUG), starting after campaign 1999, the complete transition from a carbon to a full tungsten first wall $\left[{ }^{43,44}\right]$ was monitored. Dust was collected in maintenance periods and analysed $\left[25,{ }^{45-51}\right]$ in order to observe the evolution of the dust composition after operation phases with different first wall configurations. Based on these investigations, a classification of sampled particles into dust and debris was proposed [47]. Particles which had plasma contact or were produced by direct plasma wall interaction processes are denoted as dust, whereas debris describes particles which are introduced into or produced in the vacuum vessel during maintenance work or produced during plasma operation by mechanical friction of moving invessel components. Therefore, it is important to be able to separate both, because dust is relevant for devices like ITER and beyond, while the debris can be avoided by adequate handling and can, therefore, be neglected. Unfortunately, in today's devices the fraction of debris could be significant or even dominating.

In recent studies applying analytical scanning electron microscopy (SEM with energy dispersive X-ray spectroscopy (EDX)), a link between size and outer morphology was proposed. A first evaluation of a statistically relevant number of particles from AUG was analysed regarding their composition [47-50]. For particles below $5 \mu \mathrm{m}$ in diameter, it was reported that the probability to be spherical in shape and to have tungsten as their main constituent is increased. Above $5 \mu \mathrm{m}$ in diameter, the complex conglomerates dominated by carbon and boron forming an embedding matrix for smaller $\mathrm{W}$ particles are the largest fraction of all particles [47]. Beyond these results, a more detailed classification regarding shape and composition was established in reference [49] and applied in [48,50]. The present study follows this classification scheme (see section 4). Such a classification allows the separation into safety and operationally relevant and irrelevant (e.g. debris) dust fractions. Furthermore, it could assist in elucidating production mechanisms and deliver input data for transport modelling of artificially introduced dust particles.

In this paper the strategy of dust collection studies at AUG with some historical remarks is described ranging from $\mathrm{Si}$ collector plates installed before a campaign to post-mortem collection after the campaign via adhesive tapes and the filtered vacuum sampling method (section 2 ). The paper focusses in the following on the analysis of the Si collector plates. Experimental details to the procedure used 
for obtaining and analysing a statistically relevant number of dust particles regarding outer shape and composition are given in section 3 . In section 4 the classification scheme of [49] with its adaptions [50] is presented. As one main topic of this paper, individual dust particles representing the major dust classes were selected for detailed investigation of their inner morphology. Special emphasis is placed on W-dominated spheroids and W-C-B conglomerates (section 5). Furthermore, the porosity as well as the thickness of the particles is evaluated in section $\mathbf{5}$ in order to allow a rough estimate of the dust volume and the surface area of the particles. In section $\mathbf{6}$, the statistical evaluation of the database of several 10000 particles analysed on the collectors of campaign 2009 is presented obtaining particle size probability distribution functions (PDF) for the different dust classes.

Further studies and data evaluation are necessary for a profound assessment of the overall dust content in AUG. Nevertheless, already the presented results should trigger future risk assessment studies, e.g., on the chemical reactivity of the porous conglomerate material.

\section{Dust collection strategy in AUG}

In the past, dust particles were collected in AUG by adhesive tapes as a standard procedure after each campaign and, less frequently, by filtered vacuum sampling [25]. The optical in-vessel inspection during the dust collection already indicates that much less dust is produced in AUG after the transition from $\mathrm{C}$ to $\mathrm{W}$ first wall.

When sampling with adhesive tapes, standard 3M Scotch tapes or more recently Plano-em polycarbonate tabs with $25 \mathrm{~mm}$ diameter mounted on standard SEM aluminium stubs were used. The adhesive tapes were simply stuck onto the in-vessel surfaces, after removal covered by a transparent plastic foil for storage and then mainly analysed by optical microscopy but also some by SEM.

The filtered vacuum sampling was performed using Whatman Anapore Aluminium oxide membranes with $0.02 \mu \mathrm{m}$ pore size and a membrane diameter of $47 \mathrm{~mm}$ [25]. In the 10 filtered vacuum samplings after campaign 2009, air streaming with a flow rate of $5 \mathrm{l} / \mathrm{min}$ through the nozzle (a stainless steel tube with $1 / 8$ inch diameter and 45 to $140 \mathrm{~mm}$ length) was used. The nozzle was held a short distance above the surface transporting the mobilized particles onto the filter mounted on the support grid of the stainless steel housing. The filter housing was attached to a $10 \mathrm{~m}$ long tube connected to the dry pump outside the vacuum vessel.

Both post-mortem collection techniques have the disadvantage that they collect each particle class with a different efficiency strongly depending on the class-defining particle properties, e.g., their mobilization by an air stream (expressed as aerodynamic diameter) or their sticking probability (determined by surface wetting capability of the glue of the adhesive tape). Spherical particles with a diameter less than $50 \mu \mathrm{m}$ are expected to be collected with a strongly reduced efficiency by vacuuming in contrast to adhesive tapes $\left[{ }^{52,53}\right]$. Furthermore, particles may attach to each other during filtered vacuum sampling and the outer morphology of the particles may be altered by deformation, abrasion, and break-up. Nevertheless, both techniques allow collection of dust particles on a large number of positions inside the vacuum vessel. This leads to valuable data about the local accumulation of dust and its distribution in the entire devices. Furthermore, both give an idea which types of dust and debris occur, e.g., from flakes of deposition layers over fragments of cable isolation to fibres from cloth and hairs. Therefore, all investigated horizontal areas in AUG were sampled by both techniques after the 2009 campaign leading to a unique dust collection situation. First, the vacuuming nozzle was drawn a short distance across the surface and, subsequently, the adhesive carbon tabs on standard SEM sample holders were multiply pressed with gentle force onto the surface, until the complete area was covered at least once by the carbon tab. All particles collected on these tab samples are missed by the filtered vacuum sampling, i.e., they give information about the collection efficiency and selectivity of filtered vacuum sampling. The analyses of the tab samples and filters are ongoing. Although both collection techniques are applied by different research groups on several devices (e.g. [2,1922,24,25,28,29,33]), collection efficiency assessments are missing.

Since the campaign 2006, collector plates in a specially designed stainless steel holding structure are installed in AUG. As a first try (campaign 2006), glass plates were used as collectors, but due to the 
multi-elemental composition of the glass, the element analysis using EDX was hindered. Therefore, mono-crystalline Si wafer plates have been used since 2007. As the wafers are mechanically more sensitive, the holder had to be re-constructed. The final design used since 2008 is shown in figures 1(a) and (b). The wafer is pressed to the upper part by a spring. A large contact area reduces the stress on the wafer. A box above the wafer prevents the collected dust from being removed during the vent of the vacuum vessel. An additional cap can be mounted on top of the box. This cap is removed by the last workers leaving the vessel before closing the vessel and mounted by the inspection team entering shortly after the opening. Therefore, the contamination of the wafer by debris is strongly reduced.

The advantages of this collection method are that only particles mobilized during plasma operation and not during maintenance phases arrives on the plates and that the analytical SEM analysis delivers much more reliable data due to the mono-elemental composition and the considerably flatter surface of the Si substrate compared to adhesive tapes or filters. Also only particles mobilised during a single campaign are found on the Si collectors. Furthermore, there is a high probability that all types of arriving particles stay on them. Nevertheless, also here the preselection due to the physical properties of the particles and the collector surface have to be kept in mind, i.e., size, velocity and sticking influence the collection probability of the dust particle $\left[3,{ }^{54}\right]$. Remarkably, the history of particles could be reconstructed in some cases by investigating their surroundings on the collector [45], e.g., splashing of liquid droplets and breaking off of particles. The main disadvantages of the Si wafer collector method are that the collection positions have to be selected before the experimental campaign, that only locations without strong plasma contact and low power load can be chosen, and that only a limited number of collectors can be installed.

Between 3 and 5 collectors were installed in each campaign at different poloidal and toroidal positions in AUG since 2007 (table 1) in order to obtain information about the spatial distribution of the dust particles [45,47,49]. For the campaign 2009, one collector (no. 1) on the high field side, below the heat shield, and four collectors (no. 2 to 5) at toroidal equivalent positions on the low-field side were installed. The positions of the collectors and also of the filtered vacuum sampling and adhesive tape sampling are given in the schematic cross-section of AUG in figures 1(c) and (d). Most of the data presented in this paper are obtained by investigations of the five Si collector plates from campaign 2009. First results of a statistical analysis of these five plates are given in [49] and a comparison of four successive campaigns (2007-9) for the collection on the lower PSL in sector 11 is presented in [50]. Further data evaluation of the other collector plates is ongoing.

After the campaign 2009, additional filtered vacuum sampling was performed around one of the 5 installed dust collectors and, subsequently, further dust was collected with adhesive tapes on the same area. The comparison of the results from the Si collector plates to the pair filter plus tape could lead to an overall efficiency assessment. The detailed analysis of the additionally collected 9 sample pairs, filter-tape, is still ongoing. Only a few results of the tape and filter specimens will be mentioned here.

\section{Dust analysis strategy}

In order to obtain information on the outer and inner morphology of individual dust particles and their chemical composition, the "Helios" device at IPP is used (FEI, HELIOS NanoLab 600). This device combines a SEM with a Schottky field emission electron gun and a focused ion beam (FIB) with a liquid $\mathrm{Ga}$ ion source. The inclination angle between the two beams is $52^{\circ}$. This device allows crosssectioning of dust particles with the FIB and imaging with the SEM, even during cutting. Additionally, the device is equipped with an EDX controlled by software capable of automated SEM image recording (Oxford Instruments, INCA PentaFET-x3 Si detector with "Feature" software module). The selected area (e.g., $2 \times 2 \mathrm{~mm}^{2}$ ) is segmented by the software into an appropriate number of fields. Defined by the chosen magnification, the fields had a size of about $130 \times 115 \mu \mathrm{m}^{2}$ in this study. These fields are then automatically measured, i.e., SEM images are taken, particles are detected therein by thresholding, and EDX spectra are recorded for each detected particle.

For the most frequently used magnification, the images were obtained with pixel size of $63 \times 63 \mathrm{~nm}^{2}$. Individual dust particles were identified via grey-scale to binary conversion of the field of view 
images by subsequently applying a pre-set threshold, and no further morphological image operation was performed. Be aware that the projected shape of the particles is detected. A size filter was applied, i.e., particles with an area less than a fixed number of pixels were not registered to reduce the influence of noise. The used 16-pixel-limit together with the chosen magnification for the presented analysis corresponds to an equivalent circle diameter (ECD) of $0.28 \mu \mathrm{m}$. The magnification and the pixel limit were optimised on the observed dust distribution on the AUG collectors to be able to detect the particles below $1 \mu \mathrm{m}$ while avoiding contributions from noise and the structure of the homogenously deposited film (see section 5.1).

For each detected particle, an EDX spectrum was recorded by scanning the $15 \mathrm{kV}$ electron beam across the detected particle area and elemental composition was obtained if any signal for an element, beside the ignored signal from $\mathrm{Si}$, overcomes the detection limit. With this automated SEM/EDX measurement, easily more than 10000 particles per collector can be registered and analysed during one weekend by applying $15 \mathrm{~s}$ as EDX recording time per spectrum. The beam current was restricted to $0.17 \mathrm{nA}$ in order to limit charging effects. The count rate was normally above 1000 counts per second. A searchable database including all detected particles is generated providing geometrical data (e.g., area and perimeter) and chemical composition for each particle. Note that an introduction on how to describe particles is given in [52]. Some critical remarks to image analysis and the different definitions of size and shape descriptors with their limitation can be found in [ $\left.{ }^{55,56}\right]$

The image of each field of view were additionally post-analysed by an adapted version of the freeware programme ImageJ $\left[{ }^{57}\right]$ to enlarge the number of shape descriptors for the particles and to have full access to each individual analysis step, which is quite limited in the commercial software. Furthermore, this adapted version should allow analysing images from different research groups in a comparable manner and compiling dust analysis data from different devices in an intended IAEA data base $\left[{ }^{58}\right]$.

Due to the limited size of the SEM images, large particles have a probability increasing with their size to be split onto more than one image, i.e., split into smaller particles. This splitting was manually checked for particles with one part with an area larger than $50 \mathrm{\mu m}^{2}$. Typically, less than 20 particles were split per collector on 500-1100 single images. Furthermore, particles appearing separated into particles by the automated particle detection are assigned as one, if their centre of gravity is closer together than the maximum of their Feret diameter. I.e., they are merged into one particle with an area equal of the sum of the individual particles. This treatment is aimed to eliminate erroneous splitting of a particle. On the other hand, overlapping and nearly contacting of particles have to be considered, which are correlated to the particle density. For the analysed AUG collectors, this overlapping of particles can be ignored by taking into account the fraction of area covered by particles compared to the analysed area (see Tab. 1). Be aware that overlapping is a serious problem for dust collected by filtered vacuum sampling and even adhesive tapes. The merging procedure has to apply usually about 100 times per collector to join two particles into one and less than 20 times to join more than 10 particles. However, in some cases several hundred particles are merged into one particle. The splitting into many particles is promoted by using secondary electron detection for imaging. In addition, the secondary electron detection leads to features in the images originated by charging-up, which are detected erroneously due to the fixed threshold. For these "particles" normally only Si is detected. All particles containing only Si were marked as deleted in the database. The number of deleted particles varies from 10 up to 4000 per collector of campaign 2009. The resulting database allows classification and statistical analysis of the investigated ensemble of dust particles [49] leading to size distributions functions for each class on each collector (see section 6). The final number of particles in the database per collector is given in table $\mathbf{1}$. Note that the above-mentioned posttreatments to the data were not applied in [49], but significant differences show up only on the classes "others-par" and "Cu-par" (see section 4).

For detailed analyses, higher magnified images were used. As the position of each particle is stored in the database, subsequently, many particles of each class were selected and individually imaged with higher resolution, mainly using a $5 \mathrm{kV}$ electron beam. A subgroup of these particles was then crosssectioned with FIB. Additionally, two-dimensional elemental distributions (EDX map) on some surfaces and cross-section areas were recorded in order to obtain information on the inhomogeneity of 
the particle composition. By these detailed analyses of many particles, the classification by the automated system was confirmed and, overall, no further classes had to be introduced. It was even possible to combine some classes (see section 5).

For cross-sectioning, lamella fabrication and extraction of individual particles for further analysis, a nano-manipulator system combined with the possibility of depositing platinum-carbon protection coatings with the electron or the gallium ion beam is available. Thin lamellas were made from a few particles to analyse them in transmission mode (STEM). The analysis of the inner morphology allows conclusions about the origin and history of the dust particles. Furthermore, the classification based on the outer morphology was confirmed.

\section{Classification scheme}

A classification regarding composition and shape was established in reference [49], and the present study follows this classification scheme.

The presence and absence of elements in the composition analysis of each particle is taken as the main separator. The detailed list defining the classes by presence and absence of elements in the particle composition is given in [50]. Although the filtering was only performed by presence and absence, more than $90 \%$ of the particles of a class are dominated by one element, e.g. $\mathrm{W}$.

Following the compositional analysis, some sub-sets of particles are further separated in two classes by a shape analysis regarding aspect ratio of the particle below 1.18, which represents spheroids. The aspect ratio is defined as the ratio between length to breadth of the circumferential rectangle with minimal area [52,55,56]. The separation into sub-sets of spheroids and non-spherical, irregularly shaped particles by other criteria does not strongly vary, i.e., roundness threshold of $>0.75(>0.85)$ and aspect ratio threshold of $<1.18(<1.07)$ leads to about the same sub-sets of particles.

In order to restrict the number of classes, $4 \%$ of all particles (at least for one collector), in number or area, must fall into a sub-set. So, sub-sets of all particles for each collector were built leading to classification into eight classes: tungsten-dominated (W-sph) and boron-dominated spheroids (B-sph), tungsten-dominated (W-isp), carbon-dominated (C-isp), boron-dominated irregularly shaped particles (B-isp), iron-dominated particles (Fe-par), copper-dominated particles (Cu-par), and all others which do not belong to one of the prior ones (others). Debris particles will be sorted dominantly into the later class. Even if the carbon-dominated particles are not separated in spherical and non-spherical ones, they are called C-isp. Note that the expression "flake" for the non-spherical particles as used in [49] is replaced by "irregularly shaped particles" (isp), as a flake should be thin, i.e., the ratio thickness to lateral dimensions should be small. But the thickness is not a priori known. It is for many particles even in the range of its breadth (see section 5.3). So, the expression irregularly shaped particles describes these classes better, even some particles have smooth circumferences and some others would be better described by the expression "flake" (see section 5.3). Furthermore, in order to avoid a misleading nomenclature and stick to a simple class naming scheme using the most abundant element and the dominant geometric shape the former "boron crystallites" [49] are named to boron spheroids here.

For four of the five Si collectors of campaign 2009 (see table 1) included in the further analysis (see section 5.1), about $90 \%$ of all analysed particles belong to the five main classes: $\mathrm{W}$-isp (30\%), C-isp (30\%), W-sph (20\%), B-sph, and B-isp (10\%) [49]. For the collectors of the other campaigns, this fraction is always above $85 \%$, except for two collectors. 


\section{Morphology analysis}

Before discussing the size distribution of the classes and results of the large ensemble of particles, the morphology of many particles from the main classes are individually analysed in detail and presented in this section. As a preamble, homogeneous deposition on the Si collectors must be discussed.

\subsection{Homogeneously deposited films on Si collectors}

Beside the dust particles, a surface morphology is always present on the Si collectors, which is laterally structured on the nanometre scale (tens of $\mathrm{nm}$ ). It could be expected that material is redeposited during the plasma operation in AUG covering the Si substrate and also partly the dust particles. By cross-sectioning with FIB, a layer is observed, which has a thickness of 10-30 nm for the collectors of campaign 2009, except for no. 2 and 4 of campaign 2009. For the collector no. 4, the layer is slightly thicker, $\sim 70 \mathrm{~nm}$ and for collector no. 2 it is even several hundred nanometre thick (table 1), which starts to peel off and hinders further analyses.

The chemical composition analysis shows that these thin films $(<100 \mathrm{~nm})$ contain boron and carbon and traces of iron, even if the EDX spectra are dominated by silicon and oxygen. Oxidation of the silicon substrate and the constituents of the deposited film, e.g., boron, could explained latter one. Note that the EDX sensitivity for boron is very low, i.e., only a large amount of B can be detected. Therefore, for particles coated only with a thin boron containing film, the contribution of this element is not high enough to exceed the sensitivity threshold for detection in the chosen recording time of $15 \mathrm{~s}$ in the automated EDX measurement (section 3). It is assumed that the thin films are dominantly formed during the first wall boronisations, a process depositing boron, which is routinely used in AUG for wall conditioning $\left[{ }^{59}\right]$. This assumption is supported by the largely increased thickness of the film on the collector close to the glow discharge anode used for the boronisations. Due to its thickness, the film peeled off resulting in a contrast-rich topography, which makes the automated detection of dust particles impossible [49]. Therefore, collector no. 2 of campaign 2009 as well as collector no. 4 of campaign 2008b were not taken into account for further analysis. Collector no. 4 of campaign 2009 was mounted in front of the Thomson scattering diagnostics, where in contrast to collectors no. 1, 3 and 5 of that campaign no plasma-facing components shadowed the wafer surface and thereby does not protect it from material deposition, i.e., slightly more material is deposited. The thickness of the homogeneously deposited layer only for a few collectors of the other campaigns is determined by cross-sectioning with FIB, and for all other, colorimetry $\left[{ }^{60}\right]$ was used to estimate the thickness benchmarked by the comparison to the cross-sectioned ones (table 1).

As a result, the thin deposited layer on the collectors can be ignored for the chemical composition of the particles. However, the layer has to be taken into account for the discussion of the surface morphology of dust particles, i.e., a fraction of the layer is deposited onto the particle, while the rest of the layer is found beneath the dust particle. Because each boronisation forms a new layer, a rough measure for the arrival time exists even if a minor film growth is assumed in between boronisations. That means particles on the film surface are deposited on the wafer later than particles partly or completely (dust particle diameter $<<1 \mu \mathrm{m}$ ) buried by the layer (e.g., section 5.5). This layer is assumed to act as glue and leads to a demobilization of dust particles, especially the smaller ones. Furthermore, this has to be considered for assessments of the mobilization probability and for the collection probability by adhesive tapes and filtered vacuum sampling. Note that the volume of this film is larger than the volume of the dust particles (see section 6.3).

\subsection{Cross-sectioned tungsten- and boron-dominated spherical dust particles}

The outer and inner morphology of more than hundred spherical W-dominated dust particles were analysed in more detail. The outer surface of the $\mathrm{W}$-dominated spheroids varies between smooth, bumpy with hills and groves, cracked like dried soil, to inhomogeneously composed ones with other particles attached (see figures 2-4). Three quarters of all particles in the W-sph class have a smooth surface. They appear as perfect circular from all viewing directions, i.e., they are perfect spheres, 
which allow easy and accurate calculation of their volume and surface area (see section 6.3.).

Two individual W-dominated spheroids with distinct different outer morphologies are shown in figure 2. The cross-sectioning reveals that the core of both particles is composed of solid tungsten. The thin shell on the particle shown in figures 2(a) and 2(b) is expected to originate from the homogeneous coating (see previous chapter). In contrast, the particle as shown in figures 2(c) and 2(d), which are completely covered by a shell with non-uniform thickness, supposes that the material attached during levitation of the particle. Alternatively, a stepwise coating from various sides requires frequent mobilization events. This is possible, but unlikely. Nevertheless, it is most reasonable that the attachment of material dominantly happened during particle transport through the scrape off layer plasma, as it is found for spherical particles deposited by the plasma close to the strike point $\left[{ }^{61}\right]$. Clustering processes as observed in dusty plasmas may occur $[15,16]$. Note that particle nucleation and growth in the plasma, even for tungsten, up to a few nanometre size as known from low temperature plasma $\left[15,16,{ }^{62,63}\right]$ could neither be confirmed nor disproved.

The W core of such W spheroids usually consists of several W grains with a few small pores of a size of 20-100 nm (figure 2(b)). The origin of the W spheroids is proposed to be arcing, which ejects molten tungsten solidifying in spheroid shape $\left[47,{ }^{64}\right]$. Probably, the pores are formed during the solidification process of the molten $\mathrm{W}$ droplet and the nucleation of several grains. Due to the observed grains, nucleation of smaller particles or growing from the centre as suggested for, e.g., carbon and metal dust can be excluded [18,22,29,33,62]. The perfect spherical shape indicates their solidification before arriving at the collectors. Note that the expression droplet implies that the particle was molten, while the expression spheroid classifies only the shape, here used for round particles with an aspect ratio of up to 1.18 and a smooth perimeter [49].

A negligibly small fraction of the particles of the class W-sph shows a multifaceted surface structure. EDX mapping on the cross-section of such an unusual W-dominated particle classified as W-spheroid shown in figure 3 probably reveals tungsten oxide inside and on the surface of the particle while regularly shaped boron crystallites are in between. The oxide has porous structure. The generation mechanism of that particle is unknown, although it is assumed that the process forming crystals was triggered by the air humidity after vessel opening.

Figure 4 shows another spherical particle, from which is thought that it has passed the scrape off layer plasma and which is classified as a $\mathrm{W}$-dominated spheroid, too. The spherical shape is assumed to originate from the ablation of material during transport through the plasma. It could be speculated that this particle was hot enough to emit light sufficient for detection with fast cameras [3,9]. This particle is a conglomerate of $\mathrm{W}$ particles embedded in a carbon-boron matrix (see below, [46,47,50]). Its composition is quite inhomogeneous as confirmed by detailed EDX analysis: While the bright areas are strongly dominated by $\mathrm{W}$, the dark ones contain much more boron and carbon (figure 4). Such particles are rarely attributed to the class $\mathrm{W}$ spheroids.

Most of the particles with morphology similar to the particle shown in figure $\mathbf{4}$ are assigned to the class of B-dominated spheroids. As an example, a large particle classified as B-dominated spheroid is viewed under two angles with respect to the surface in figure 5 . Note, by far most of the B-dominated spheroids are smaller than $1 \mu \mathrm{m}$ (see section 6.3.). The height of such particles measured in crosssections is found to be approximately equal to their diameter (i.e. ECD). Here, no significant porosity is detected by cross-sectioning.

From the general evaluation of the class of $\mathrm{W}$-dominated spheroids, the particles are determined to be perfect solid W spheres with ECD as diameter and with negligible porosity. The B-dominated spheroids are proved to be spherical, too. Many of those are composed of a mixture of $\mathrm{W}$ particles in a B matrix, similar to some $\mathrm{W}$-dominated spheroids.

Considering the amount of smooth $\mathrm{W}$ spheres and the fraction of particles showing coatings and homogeneously distributed, attached smaller particles around a $\mathrm{W}$ core, more than $90 \%$ of all $\mathrm{W}$ dominated spheroids are W droplets, at least for campaign 2009. The W spheroids are found all around the torus as first analyses of the tape specimens confirm. The $\mathrm{W}$ droplets are expected to be produced by arcing (see section 5.4). A minority could be produced by macroscopic melting events in AUG, accidental and intentional ones $\left.{ }^{[5,66}\right]$. Melting of $\mathrm{W}$ particles during the levitation phase could 
occur if they come close to the separatrix. But if they travel only in the far scape off layer plasma, melting of $\mathrm{W}$ is unlikely $\left[3,62,{ }^{67}\right]$, while partial melting of the B-dominated particles is more likely due to a melting point of boron-containing phases below $3000 \mathrm{~K}$. If they were molten, various compound phases and solidification features should be observable by detailed future analysis (see phase diagrams of the compounds of $\mathrm{W}, \mathrm{B}$, and $\mathrm{C}\left[{ }^{68-70}\right]$ ). However, the spherical shape of the dust particles alone is not an indicator for melting. The spherical shape could also be caused by strong ablation of material during travel through the scrape off layer plasma.

The analyses of the dust collected on $C$ tapes and filters confirm the presence of the same type of spherical particles with comparable outer morphology.

\subsection{Tungsten, boron- and carbon-dominated irregularly shaped particles}

In figures 6 and 7, particles of the three classes of irregularly shaped particles (W-isp, C-isp and B-isp) are shown. One carbon-dominated irregularly shaped particle is presented in the figures 6(a) and 6(b) under two viewing angles revealing the flatness of this particle. Furthermore, the cross-sectioned part of the particle in figure $\mathbf{6}(\mathbf{b})$, marked by arrows, clearly demonstrates the high porosity of this flake. The pores cover $\sim 30 \%$ of the cross-section area. The composition of the particle of figure $\mathbf{6}(\mathbf{a})$ is quite inhomogeneous, which is very similar to composition variations observed on the spherical conglomerates (figures 4, 5). The figures 6(c) and 6(d) show a small B-dominated irregularly shaped particle, which sticks in the homogeneously deposited film from AUG (section 5.1). Clearly, the film partly covers the particle and is thinner below than beside it (figure 6(d)). In addition, this particle has a pore. The W-dominated irregularly shaped particle shown in figure 6(e) and 6(f) is quite dense while the $\mathrm{W}$-isp in figure 7 has even a porosity far over $50 \%$. The later one has more a sponge-like structure with some similarity to the C-dominated particle shown in figure 6(a) and 6(b).

The analysis of many W-, C-, and B-dominated irregularly shaped particles - also on the C tape collectors - leads to the conclusion that a significant fraction of them consists of the same material conglomerate, which only differs in the amount of each element, i.e., the number and size of embedded $\mathrm{W}$ particle in a B-C matrix. Therefore, it could be suggested to join for further analysis (section 6) the three irregularly shaped particle classes and the B-spheroids as they consist all of the conglomerate material and call them further on WCB-isp. Note that iron is usually found as a trace impurity in many of these particles, if their EDX spectrum is recorded with sufficient statistics, i.e., much longer acquisition time than used during the automated particle detection (section 3).

Many conglomerate particles look very fragile, e.g., the particle in figure 7, indicating that they did not have intense contact to plasma.

The same conglomerate material in particles should have the same origin: re-deposited material, which flaked away, e.g., by thermal stresses during arcs, transient power loads and disruptions (see references $[47,48])$.

For many irregularly shaped particles their thickness was determined by cross-sectioning (figure 6(b,d)), by stereometry, i.e., by viewing the same particle under different angles by tilting (figure 5(a,b)) and by analysing the shadow created by scanning with the FIB (figure $\mathbf{6}(\mathbf{f})$ ). Taking the observed thickness, the length $l$ and breadth $b(l>b)$ as well as the porosity of investigated particles of each class into account, the volume of the particle of these three classes could be roughly assessed by a three-axial ellipsoid with a correlated thickness $t$ to the breadth $b$ of $t=\tau \cdot b$ (section 6.3). The thickness of the most particles is below the breadth, in the range between $20 \%$ and $100 \%$ of the breadth, with lower values for larger particles. The constant $\tau$ is roughly estimated to be 0.3 for the three classes of irregularly shaped particles taking the thickness and the observed shape into account. Note that for one fixed constant, the volume of larger particle will be in average overestimated while for smaller ones it is underestimated. As a lower limit for the surface area, the half of cuboid surface with $l, b$, and $t$ is taken. In order to take the surface roughness and the porosity into account and therefore the real surface area, the geometrical surface area must be multiplied by a factor $\eta$, which could be estimated be to up to 1000 , while a more realistically factor $\eta$ is between 10 and 100 . 


\subsection{Origin of conglomerate material and tungsten droplets}

Figure 8 shows the surface of AUG divertor tile that suffered strong arcing activity. The arcs burned through the W-coating layer into the graphite substrate $\left[64,{ }^{71,72}\right]$, which is observable as dark areas in figure 8(a). The arcs splash away tungsten leading to $\mathrm{W}$ droplets $\left[{ }^{73}\right]$, e.g., even visible on the graphite area close by in figures 8(b)-(c)). Parallel to this obviously most recent arc track in the middle and in

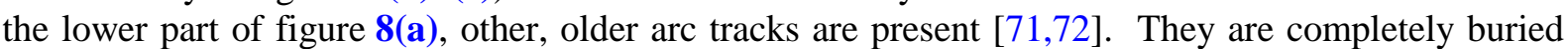
under re-deposited material, which also covers the areas between the arcs. This material is composed by subsequent deposition from the plasma and arcing activity in its vicinity, which splashed molten and evaporated material. Partly this material is very porous after venting (figures $\mathbf{8 ( d )}$ ). If an arc burns on this porous, layered and thick re-deposited material, it could melt (figure 8(c)) and crack away (figure 8(b)) as conglomerated material. In the vicinity of the arc tracks, flakes of this material are observed which lay loosely on the tile surface.

The surface morphology of this conglomerated, flaked, and partly molten material (figure 8) is equal to W-, C-, and B-dominated irregularly shaped particles (figures 6 and 7). In the vicinity of the arc, temperatures above the melting point of $\mathrm{W}$ are reached, which is higher than the melting points of the compounds [68-70]. So, melting and segregation effects are expected. It is assumed that a large fraction of this conglomerate material flaked off arrives at the collectors without plasma contact, as the fragile structure observed on many individual particles indicates (figures 6 and 7), while some other fraction passes the plasma without complete ablation and leading to the formation of such spheroids as shown in figures 4 and $\mathbf{5}$. The fraction of starting particles, conglomerates and droplets, which is completely dissolved by plasma contact, is unknown. Note that only particles, which the plasma heated and kept at sufficient high temperatures [3] and which are sufficiently large to survive long enough, could be detected by fast framing cameras [9]. The detection limit for observing with fast cameras is under discussion and is in the range of 1 and $10 \mu \mathrm{m}[9,10,11,36]$.

Furthermore, the molten $\mathrm{W}$ ejected by the moving arcs are found on this tile in and beside the arc tracks as droplets as well as everywhere in the device, i.e., on the dust collectors (see section 5.2).

\section{Statistical size distribution analysis}

For most classes, the number of particles in the class is large enough to be statistically relevant. Therefore, the evaluation of their size distribution is performed. In the following, the ECD is taken as a measure for the particle size. Note that the ECD is correlated to the area, which is the most robust particle size descriptor [52].

\subsection{Lognormal, power law and Weibull probability density function}

The sizes of dust particles are presumed to follow a certain continuous probability distribution. Historically, measurements of dust particle sizes from tokamaks have been presented alongside a fitted probability density function (PDF), the form of which has been assumed a priori. This has often been a lognormal distribution (see e.g. [2]), which commonly applies to solid particle size distributions $\left[{ }^{74}\right]$. More recently, a power law distribution has been suggested [29], as already used earlier for particles originated by arcs [73]. Both distributions may provide a good fit for dust particles [27].

The difficulty in distinguishing these distributions has been discussed at length in reference $\left[{ }^{75}\right]$. Power law behaviour is often assumed when data, plotted as a number of particles per size bin, appears roughly linear on a log-log plot. Linear regression then may be applied to determine the power, and the resulting $R^{2}$ value may be taken as a measure of the quality of the fit. However, the formulas of linear regression do not apply if the data are transformed in this way, and the linear regression can lead to poor fits even for a high $R^{2}$ value. Furthermore, it cannot account for data sets that are truncated, for example by measurement limitations. Failure to account for this can lead to an incorrect determination of the particle size distribution, and can lead to rather different conclusions about the surface area and mass distributions, which has been demonstrated in reference $\left[{ }^{76}\right]$. 
Another distribution, the Rosin-Rammler distribution, was introduced nearly 100 years ago to describe the size distribution of particles $\left.{ }^{77}\right]$. It has the same form [74] as the Weibull distribution; the latter includes the simple exponential $(\mathrm{k}=1)$ as well as the Rayleigh distribution $(\mathrm{k}=2)$ as special cases.

In this paper three probability density functions, lognormal, power law, and Weibull, are tested to fit the experimentally obtained particle size distributions. The PDF gives the probability of finding a particle in a given size range. Thus, it integrates to 1 on the interval $[0, \infty[$. Due to technical reasons, the data are unreliable below a certain size, $d_{\min }(0.28 \mu \mathrm{m}$ in this study). In addition, an upper limit exists, $d_{\max }$, e.g., the incircle diameter of the analysed area. The differences of these two limits depend on their relation to the distribution, i.e., to the parameters arithmetic and geometrical mean, median, and mode. For all distributions here, $d_{\max }$ is far away from these parameters, while $d_{\min }$ is close to them, i.e., only a lower truncation has to be taken into account. Therefore, the PDFs must be renormalized such that they integrate to 1 on the interval $\left[d_{\min }, \infty[\right.$. Such truncated distributions must be used to fit the data and are given for the lognormal, power law, and Weibull distribution, respectively:

$$
\begin{aligned}
& f_{\text {Lognormal }}(d)=\frac{1}{\frac{1}{2} \operatorname{erfc}\left(X\left(d_{\text {min }}\right)\right) \cdot d \cdot \sqrt{2 \pi} \cdot \ln (s)} \cdot \exp \left(-(X(d))^{2}\right) \\
& \text { with } X(u)=\frac{\ln (u)-\ln \left(d_{g}\right)}{\sqrt{2} \cdot \ln (s)} \\
& f_{\text {Power-law }}(d)=\frac{\alpha-1}{d_{\text {min }}} \cdot\left(\frac{d}{d_{\text {min }}}\right)^{-\alpha} \\
& f_{\text {Weibull }}(d)=c_{W} \cdot \frac{k}{\lambda} \cdot\left(\frac{d}{\lambda}\right)^{k-1} \cdot \exp \left(-\left(\frac{d}{\lambda}\right)^{k}\right) \quad \text { with } \quad c_{W}=\exp \left(\frac{d_{\text {min }}}{\lambda}\right)^{k}
\end{aligned}
$$

Here $d$ is the variable diameter (i.e., ECD), $d_{\min }$ is the lower measurement limit, $d_{\mathrm{g}}, s, \alpha$, and $\lambda$ are fit parameters and $\operatorname{erfc}(x)$ is the complementary Gauss error function. For the lognormal distribution, $d_{\mathrm{g}}$ represents the geometric mean, and $s$ the geometric standard deviation. (Note that often $\mu=\ln \left(d_{\mathrm{g}}\right)$ and $\sigma=\ln (s)$ are used as parameters.)

Integrating the PDFs from $d_{\min }$ to $d$ results in the cumulative distribution function (CDF) for the lognormal, power law, and Weibull distribution, respectively:

$$
\begin{aligned}
& F_{\text {Lognormal }}(d)=1-\frac{\operatorname{erfc}(X(d))}{\operatorname{erfc}\left(X\left(d_{\text {min }}\right)\right)} \\
& F_{\text {Power-law }}(d)=1-\left(\frac{d}{d_{\text {min }}}\right)^{1-\alpha} \\
& F_{\text {Weibull }}(d)=1-\exp \left(\left(d_{\text {min }} / \lambda\right)^{k}-(d / \lambda)^{k}\right)
\end{aligned}
$$

For a given value of $d$, the undersize CDF gives the probability that a sampled particle will be smaller than that size; it approaches one as $d$ goes to infinity [52]. Note that the CDF has a value of 0.5 for the median size of the particle distribution.

The fitting of the ensembles of the individual particles is performed using maximum likelihood estimation in programming language $R\left[^{78}\right]$, and the quality of fit judged by the Kolmogorov-Smirnov (KS) statistic, as recommended in [75]. The KS statistic, $D_{K S}$, is simply a measure of the maximum difference between the CDF obtained from the data $\left(F_{d}\right)$ and the fit $\left(F_{f}\right)$ at any value of $d$ :

$$
D=\max \left|F_{d}(d)-F_{f}(d)\right|
$$


A good fit is indicated by a small value of $D_{K S}$.

Unlike the power-low and the Weibull distribution, the lognormal distribution allows the extrapolation to zero and arbitrarily large particle sizes. For the lognormal distribution, the transformation from $d$ to volume $V$ of a sphere $\left(V=\pi / 6 \cdot d^{3}\right)$ taking into account the transformation of the volume elements of the two parameters results in the CDF for the volume $V$ :

$$
F_{\text {Lognormal-Vol }}(V(d))=\frac{1}{2} \operatorname{erfc}\left(-\frac{\frac{1}{3} \ln \left(d^{3}\right)-\ln \left(d_{g}\right)}{\sqrt{2} \cdot \ln (s)}\right)
$$

and the expectation value of the volume $\langle V\rangle$ :

$$
\langle V\rangle=\frac{\pi}{6}\left(d_{g}\right)^{3} \cdot \exp \left(\frac{9}{2} \cdot(\ln s)^{2}\right)
$$

Therefore, the total volume $V$, i.e. mass, of a particle class can be determined, when the size distribution is fitted by a lognormal distribution and the observed particle density of the fitted particle ensemble is known. Integrating the volume using the distribution from zero to a critical diameter $d_{c}$ leads to:

$$
I_{V o l}\left(d_{c}\right)=\frac{\langle V\rangle}{2} \operatorname{erfc}\left(-\frac{\ln \left(d_{c}\right)-\ln \left(d_{g}\right)-3(\ln (s))^{3}}{\sqrt{2} \cdot \ln (s)}\right)
$$

With this equation, upper particle size limit can be determined for which, e.g., $75 \%$ of the total volume of the particle ensemble is in particles with lower size $\left(d_{75 \% \mathrm{vol}}\right)$. Note that this upper limit allows to judge if the chosen magnification used in the SEM analyses is adequate for the investigated particle ensemble. Furthermore, the fraction of the total volume present in the particles with a size below the detection limit, $\mathrm{d}_{\min }$, can be calculated.

For a particle density $N_{\text {ensemble }}$ of each ensemble (dust particle class), the total volume $V_{\text {total-Lognormal }}$ for the lognormal distribution can be calculated by:

$$
V_{\text {total-Lognormal }}=\frac{N_{\text {ensemble }}}{\frac{1}{2} \operatorname{erfc}\left(X\left(d_{\text {min }}\right)\right)}\langle V\rangle,
$$

where the denominator takes into account the unobserved particles with diameter below $d_{\text {min }}$. Therefore, the total particle volume for each fitted ensemble can be extrapolated from the truncated data set using the fit parameter ( $d_{\mathrm{g}}$ and $s$ ) as well as the fraction of the total volume missed in the measurements (small and large particles) can be given. However, extrapolation should always be done with caution checking the contribution from the extrapolated part.

\subsection{Dust particle size distributions on the collectors}

The particle size distribution of all eight classes (see section 4) from each collector of campaign 2009 is separately used as input data for the fitting as well as some merged data sets: i) the data from collectors no. 3, 4 and 5 due to their poloidal equivalent mounting position on the low field side, ii) the three classes of irregularly shaped particles plus the class of boron-dominated spheroids due to the similarity of their composition and inner morphology (WCB-isp, see section $\mathbf{5 . 2}$ and 5.3) as well as iii) all particles from all collectors of the campaign 2009, except from collector 2 (see section 5.1). For displaying measured data, each particle ensemble is divided into 30 bins with exponentially increasing width. For obtaining the same units as the PDF, the binned counts are divided by the bin width and are normalized to the total sample size. Some of the fit parameters and KS statistics are summarized in table 2. It must be stressed that the fit parameters were obtained by maximum likelihood estimation based on the entire sample of particles, not the binned data.

As has been observed in other devices [27,29], some of the data indeed look more or less like a power law. For example, the distribution of carbon particles on collector no. 5 is shown on a log-log plot in 
figure 9(a). Despite the appearance of an approximate power law in some of the samples (figure 9(a)) or a simple exponential behaviour of some other samples (e.g., figure 9(b)), the fits to each analysed distribution and associated KS test reveal that a truncated lognormal distribution is the better fit in most of the 48 fitted ensembles. Figure $\mathbf{1 0}$ shows in linear scale the size distribution of $\mathrm{W}$ spheroids from the low field side. For illustrating the high reliability to describe the data by the lognormal fitting, the insert in figure $\mathbf{1 0}$ shows the contour plot of the logarithm of the likelihood in the space of $d_{g}$ and $s$. It has a very sharp maximum around the values given in table $\mathbf{2}$ indicating the very accurate description of the data by the lognormal distribution. By taking randomly 1000 pairs of $d_{g}$ and $s$, weighted by their likelihood, the $90 \%$ confidence interval can be defined, which is plotted together with their corresponding PDFs in figure $\mathbf{1 0}$ with linear particle size scales.

In figure 11 the lognormal fits are shown on a log-log plot together with the measured data for the $\mathrm{W}$ spheroids and the sum of all three classes of irregularly shaped particles plus the B-spheroids, WCBisp, for the low field side, i.e., collectors no. 3, 4 and 5, and for the high-field side, i.e., collector no. 1. The usual lognormal shape for the size distribution is only apparent for the W-spheroids of the low field side, less pronounced for the $\mathrm{W}$-spheroids of the high field side. On the other hand, the irregularly shaped particles occur with increasing probability as $d$ approaches $d_{\min }$. In these cases, it is only the tail of the lognormal distribution that describes the data and is fitted; the maximum of the curve is below $d_{\text {min }}$. These fits for irregularly shaped particles are characterized by a smaller geometric mean $d_{g}$ and larger geometric standard deviation $s$ than the $\mathrm{W}$-sph, as it is evident from table 2 . Despite that the geometric mean $d_{g}$ and standard deviation $s$ for the individual classes of the collectors no. 3, 4 and 5 vary up to a factor of around 2, from the optical impression no differences are obvious from comparing the binned distributions of the individual collectors. Furthermore, the size distributions of irregularly shaped particles, WCB-isp, are about the same for the low and the high field side, i.e., their distribution is not affected by position of the collector in the torus.

For the $\mathrm{W}$ spheres, a poloidal variation is present leading to a factor of 2 larger geometrical mean and median size for the low field side (figure 11). In addition, the small deviation of the binned data from the lognormal distribution at low ECD is present in each single distribution of the $\mathrm{W}$ spheroids. This systematic deviation could be explained by the erroneously classified small irregularly shaped particles as spheroids. Recall that the tungsten spheres and irregularly shaped particles have been distinguished by their aspect ratio, the ratio of length to breadth. The separation into spheroids and irregularly shaped particles is sensitive to the threshold value for the aspect ratio and, therefore, the maximum of the size distribution is affected. For example, using 1.07 instead of 1.18 as threshold for the aspect ratio leads to an increase of the maximum in the size distribution from $\sim 1 \mu \mathrm{m}$ to $\sim 2 \mu \mathrm{m}$ [50] for the low field side. Also for the high field side, the maximum shifts to larger values.

\subsection{Volume, mass and surface area assessment of dust particle}

The volume, mass and the surface area of the dust particles are of importance for the safety assessments. The particle volumes $(V)$ and surfaces $(S)$ for the particle ensembles discussed in section 6.2 have been calculated directly from the individual particles using the estimates given in section 5.2 and 5.3. The volume and surface of the tungsten and boron spheroids are calculated from the diameters by $V_{s p h}=\pi / 6 \cdot E C D^{3}$ and $S_{s p h}=\pi \cdot E C D^{2}$, while for the tungsten-, carbon-, and borondominated irregularly shaped particles, WCB-isp, a three axial ellipsoid $\left(V_{i s p}=\pi / 6 \cdot l \cdot b \cdot t\right)$ and cuboid $\left(S_{\text {isp }}=0.5 \cdot 2 \cdot(l \cdot b+l \cdot t+b \cdot t) \cdot \eta\right)$ are used. Here $l$ is the particle length, $b$ the breadth and $t$ the thickness which is estimated to be correlated to the breadth $b$ by $t=\tau \cdot b$ with $\tau=0.3$. The real surface area $S_{\text {isp }}$ is obtained by inserting a realistic value for the factor $\eta$ of 10-100 (see section 5.3). The resulting volume and surface area values for W-sph and WCB-isp and high and low field side by summing up all individual particles and scaled to the analysed area are given in table 3 (using $\eta=100$ ). These values underestimate the real value because the measured data are truncated on both sides, but in particular for the large particles (see table 3 for the largest particle present in each ensemble). This mismatch is larger for the irregularly shaped particles, because the distribution is broader, i.e., larger particles are less included in the analysed particles. Further on, the volume can be transformed in mass by assuming mass densities for the different classes of particles, e.g., W bulk density of 
$19.3 \mathrm{~g} / \mathrm{cm}^{3}$ for the W-spheroids, while $3 \mathrm{~g} / \mathrm{cm}^{3}$ in average for WCB-isp (see table 3), taking also porosity into account.

Taking advantage of the knowledge of the size distribution, some quantities of interest can be extrapolated, which are given for some ensembles in table 3 . The particle size $d_{75 \% \text { vol }}\left(d_{90 \% \text { vol }}\right)$ can be calculated with equation (10), for which less than $75 \%$ (90\%) of the volume of the ensemble is contained in particles up to the size $d_{75 \% \mathrm{vol}}\left(d_{90 \% \mathrm{vol}}\right)$. In addition, the fraction $R_{d}$ of all particles with ECD below $d_{\min }$ and fraction $R_{\text {vol }}$ of the total volume due to particles with ECD below $d_{\min }$ can be determined (equations (4) and (10)). Note that the irregularly shaped particles are fitted by one size parameter, ECD, even if for the volume and surface area of the experimental data two other individual size parameters are used. However, this is justified because the aspect ratio, length to breadth, is below 1.5 and 2 for more than $70 \%$ and $90 \%$ of these particles, respectively. Furthermore, the total dust volume $V_{\text {total-Lognormal }}$, i.e., total dust mass per area is extrapolated from the lognormal fit (equations (11)). For the mass of the WCB-isp, the volume is reduced by the factor $\tau=0.3$ (see section 5.3), i.e., flattened spheres.

For all fitted ensembles (see table 3 ), $d_{75 \% \text { vol }}$ is about equal to the largest observed particle of the ensemble, indicating the appropriate choice of the analysing parameters (section 3). For the fraction $R_{d}$ for $\mathrm{W}$-spheres on the low field side, only $1 \%$ of cumulative volume is not described by experimental data, while for some other classes the fraction $R_{d}$ even reaches $50 \%$, i.e., leading to larger error if taking only the experimental data. However, the fraction $R_{v o l}$ is far below $1 \%$ for all ensembles, i.e., the truncation of the small particles does not affect the total volume obtained from the experimental data. For the $\mathrm{W}$ spheres, the total dust masses obtained directly for the experimental data and from the extrapolation from the lognormal fit are about the same, while for the WCB-isp they differ only by a factor of around 2 for the different ensembles. This can still be accounted as very good agreement, especially when remember that the WCB-isp are only fitted by flattened spheres.

Finally, such data as given in table 3 allow to estimate the total dust mass arrived at the roughly $5 \mathrm{~m}^{2}$ horizontal area in AUG, for which the Si collectors are representative $\left(0.7 \mathrm{~m}^{2}\right.$ for high field side and $4.1 \mathrm{~m}^{2}$ for the low field side). The variation of this estimated total dust mass of the eight dust classes (section 4) on the different collectors of campaign 2009 is less than a factor 3. For the four ensembles in table 3 the estimated total dust mass is given. It follows that altogether less than $300 \mathrm{mg}$ arrived on areas represented by the Si collectors during campaign 2009 in AUG. The overwhelming part of these $<300 \mathrm{mg}$ is found on the low field side, and both, the W-sph and the WCB-isp, contribute about equal to it.

\section{Conclusion}

The dust collection strategy in AUG allows acquisition of statistically relevant data building a solid basis for the development of a classification scheme of dust produced by plasma-wall-interaction [49]. This scheme allows separating debris from dust produced during the plasma operation. By analysing the inner morphology of individual particles of each class, this scheme is refined and confirmed. The present analysis focuses on the W-dominated spheroids and the W-, C-, and B-dominated irregularly shaped particles, which are together about $90 \%$ of all analysed particles, in number, covered area as well as in volume.

More than $90 \%$ of all W-dominated spheroids are W-droplets presumably originating from arcs. Furthermore, at least about a quarter of all W-dominated spheroids interacted with plasma, e.g., coming close the separatrix. Nevertheless, only a small fraction of them, the largest and hottest, is potentially observable by fast cameras. Altogether, it is reasonable to generalize the properties of all particles of the class "W-dominated spheroids" for safety assessment as ideal spheres composed only of $\mathrm{W}$, i.e., minimal surface area to volume ratio at high density.

On the other hand, the irregularly shaped particles show a considerably larger surface area to volume ratio for lower density material. As a first result, a large part of all irregularly shaped particles are conglomerates of $\mathrm{W}$ embedded in a C-B matrix possibly produced by flaking off of deposited films from areas with strong arcing activity. Note that the thickness of deposited films in AUG is strongly 
reduced since switching to a full $\mathrm{W}$ first wall $\left[{ }^{79}\right]$. The conglomerate particles exhibit a porosity of even above 50\%. Furthermore, their partly fragile structure leads to the assumption that at least a larger fraction of conglomerate particles did not have strong interaction with plasma before arriving at the collectors, i.e., these particles are not observable by video cameras and not affecting the plasma performance. The porosity and the irregular shape with a huge surface area points to a very high chemical reactivity in the case of accidents, i.e., they bear a high safety risk. In spite of porosity and irregular shape, the volume of the class of W-, C-, and B-dominated irregularly shaped particles is roughly assessed by an ellipsoid. Beside the measured length and breadth, the height is determined to be in average $30 \%$ of the breadth.

The analysis of the particle size distribution indicates that at AUG dust follow a lognormal distribution. By measuring a sufficient part of the size distribution, the total volume, i.e., the total mass, of the dust can be extrapolated. For the campaign 2009, less than $300 \mathrm{mg}$ of W-containing dust is deposited on the $5 \mathrm{~m}^{2}$ horizontal areas of AUG.

\section{Acknowledgment}

This work, supported by the European Community under the contract of the EURATOM Association was partly carried out within the framework of the EFDA Task Force on Plasma Wall Interactions. The views and opinions expressed herein do not necessarily reflect those of the European Commission.

\section{Figures}

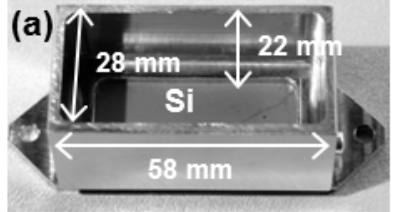

(c)

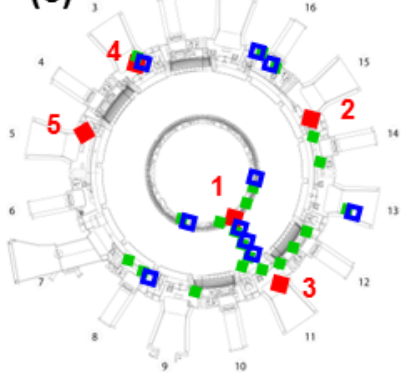

(b)

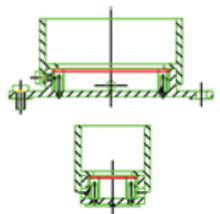

(d)

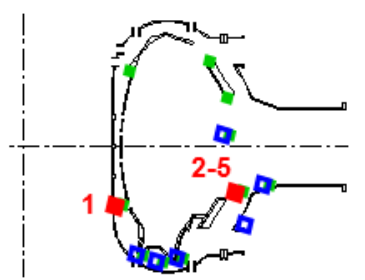

Figure 1 (one column figure): (a) Photograph and (b) technical drawing of the stainless steel dust collector housings used since campaign 2008b in AUG: The position of the silicon wafer is indicated by the diffusively reflecting plate (photo), which is pressed against the metallic frame from the bottom side. Schematic drawings of (c) poloidal and (d) toroidal cross-section of AUG: For the dust collection during and after the campaign 2009, the positions of the Si-collectors are indicated by 5 numbered big red squares while the filtered vacuum sampling positions by open squares and the tape sampling positions by small green squares. 

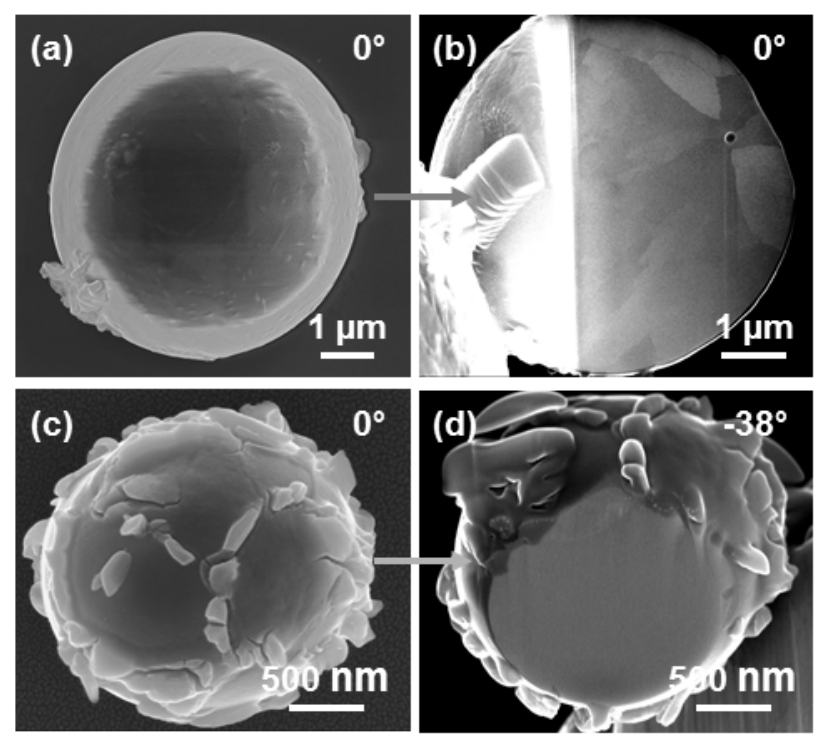

Figure 2 (one column figure): (a) Top view SEM image of a $\mathrm{W}$ droplet of $\sim 6 \mu \mathrm{m}$ in diameter with some surface roughness and attached material (no. 1, campaign 2007). (b) The same particle as shown in (a) after transferring it onto a Cu TEM-grid (left border) and thinning its right half to electron transparency $\left(E_{p}=30 \mathrm{keV}\right)$. The internal $\mathrm{W}$ grain structure after solidification and a thin layer at the outer edge is clearly visible. The cross-section is viewed under angle of $0^{\circ}$ (STEM geometry). (c) Top view of a $\mathrm{W}$ droplet of $\sim 2 \mu \mathrm{m}$ in diameter with some attached material and cracks on the surface (no. 1, campaign 2007). (d) The same particle as shown in (c) after transferring it onto a Cu TEM-grid (right lower corner) and cross-sectioning. The cross-section is viewed under angle of $-38^{\circ}$. The outer morphology of the particle is altered by Ga ion impact, Pt-C-coating, and redeposited material from the FIB processing.
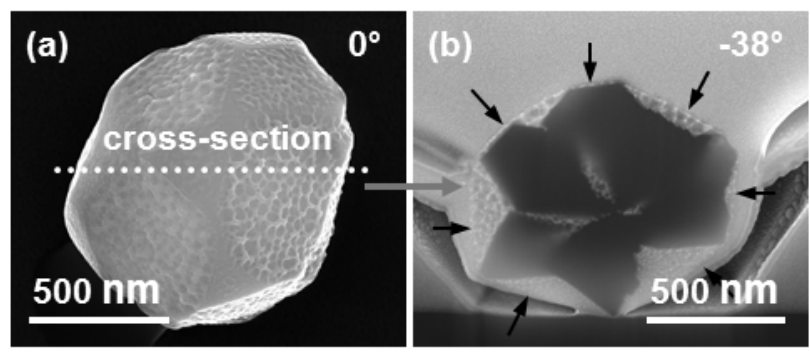

Figure 3 (one column figure): (a) Top view SEM image of a nearly spherical particle with a multifaceted surface and a porous surface sub-structure, classified automatically a W-dominated particle. (b) Cross-section image of the particle of (a) after covering with a Pt-C protection coating (bright part). The Si collector plate (no. 4, campaign 2008b) is visible (dark, lower border of the image), while the boron-dominated core (dark star-like structure) is surrounded by tungsten oxide visible as porous structure in (a). The black arrows mark the boundary of the particle, and the large pores outside the particles are artefacts of the Pt-C coating. The cross-section is viewed under angle of $-38^{\circ}$. 

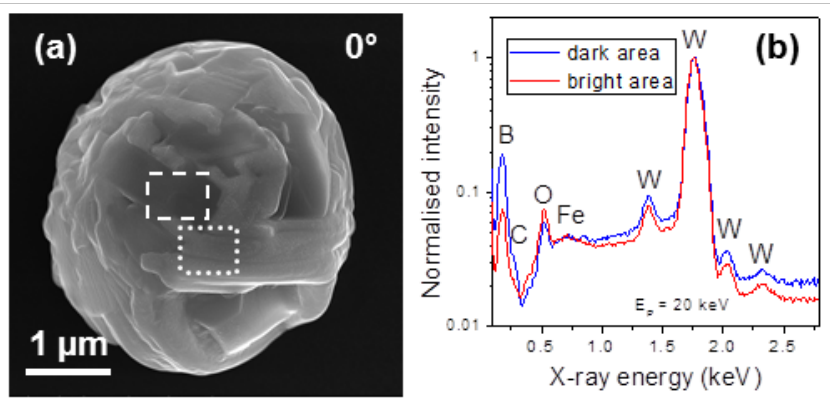

Figure 4 (one column figure): (a) Top view SEM image of a large W-dominated particle of about $8 \mu \mathrm{m}$ in diameter with some dark and bright area (no. 4, campaign 2008b). (b) EDX spectra of the two marked areas are normalised to the main W peak $(1.76 \mathrm{keV})$. Clearly, the strong increase of the boron signal for the dark region is visible. The signal of $\mathrm{W}$ in the dark area is explained by the penetration and spreading of the $20 \mathrm{keV}$ primary electron energy, $E_{p}$.
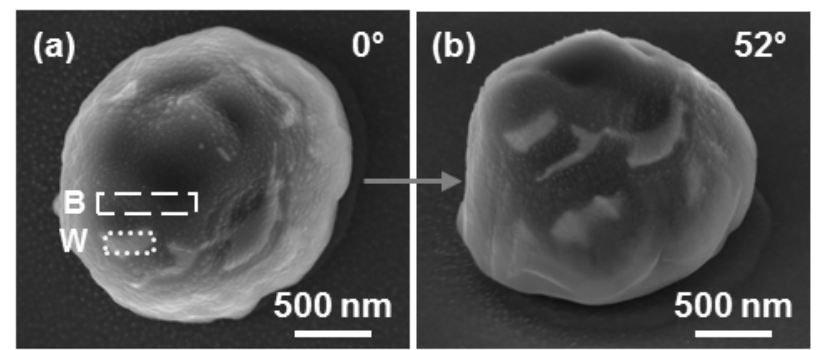

(c)

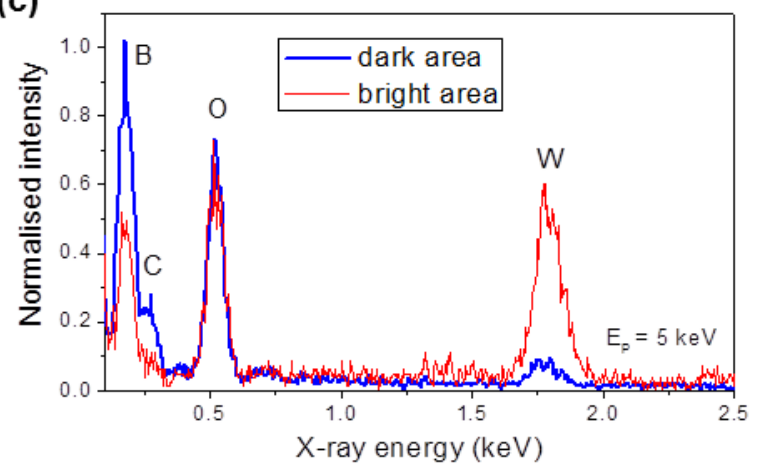

Figure 5 (one column figure): (a) Top view SEM image of a B-dominated spheroid of about $1.5 \mu \mathrm{m}$ in diameter with some dark and bright areas (no. 4, campaign 2009). (b) Same particle viewed after tilting by $52^{\circ}$. (c) EDX spectra normalised to the measurement duration of the two marked areas in (a) are obtained with $5 \mathrm{keV}$ primary electron energy, $\mathrm{E}_{\mathrm{p}}$. 

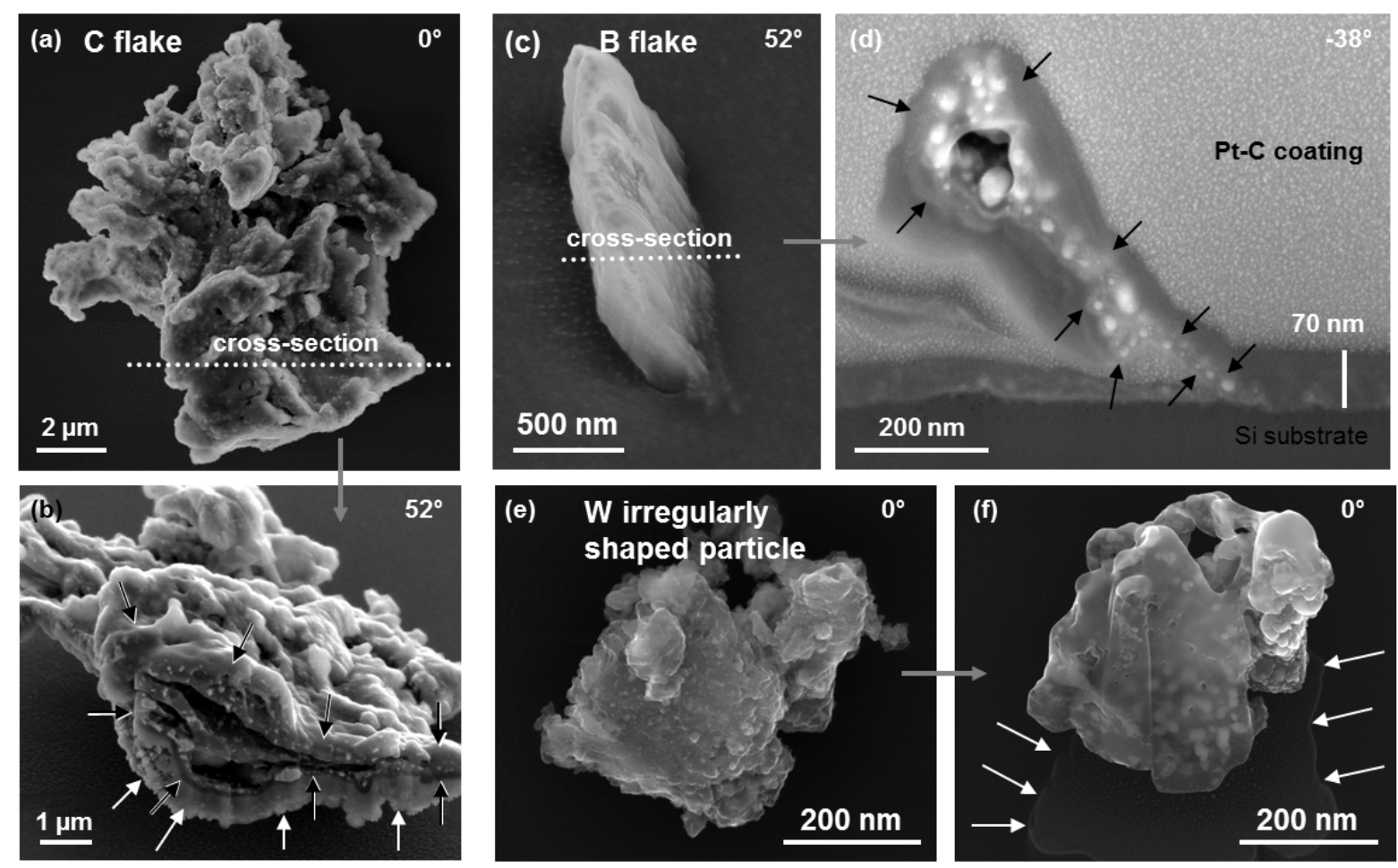

Figure 6 (two column figure): Examples of the three types of irregularly shaped particles on the $\mathrm{Si}$ collectors no. 4, campaign 2009 (a-d) and no. 2, campaign 2008b (e,f): (a) Top view SEM image of a $\mathrm{C}$-dominated conglomerate flake and (b) the same particle partly cross-sectioned after tilting by $52^{\circ}$ (i.e. surface is viewed under $52^{\circ}$, while the cross-section under $-38^{\circ}$ ). The black arrows in (b) indicate the cross-sectioned area of the particle at the position marked in (a). The layer below the particle in the cross-section in (b) is artificially redeposited material during the FIB cutting (marked by white arrows). (c) SEM image of a B-dominated conglomerate flake viewed under $52^{\circ}$ to the surface normal and (d) cross-section at the position indicated in (c) after imbedding the particle in Pt-C coating viewed under $-38^{\circ}$. The thickness of homogeneous deposition of the collector by redeposited material during the plasma operation and boronisation is indicated in (d). The black arrows in (d) mark the edge of the particle, which sticks in the redeposited material. (e) SEM image of a W-dominated irregularly shaped conglomerate particle viewed under $0^{\circ}$ and (f) after cross-sectioning without tilting (i.e. the cross-section area is viewed under $\left.-38^{\circ}\right)$. The shadow of the particle on the Si substrate due to the scanning with the FIB (lower part in (f), marked with white arrows) allows an estimation of the particle height, which is in this case $\sim 500 \mathrm{~nm}$. Note that the $30 \mathrm{keV}$ Ga ion beam damages the fragile structure of this particle, as can be seen from the comparison of the particle surface in the images (e) and (f), e.g., top part of the particle. This damaging is also visible on the particle surface in (b). 

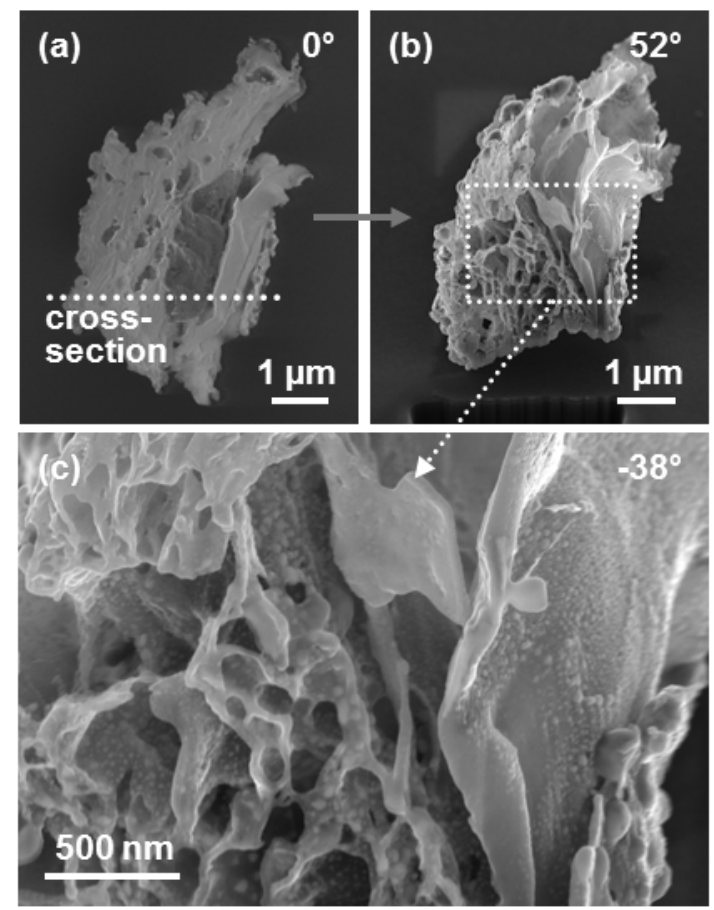

Figure 7 (one column figure): (a) Top view SEM image of a W-dominated irregularly shaped conglomerate particle and (b) after cross-sectioning after tilting by $52^{\circ}$. The cross-section area is viewed under angle of $-38^{\circ}$. (c) Cross-sectioned area magnified viewed under $-38^{\circ}$ indicates porosity far above $50 \%$.
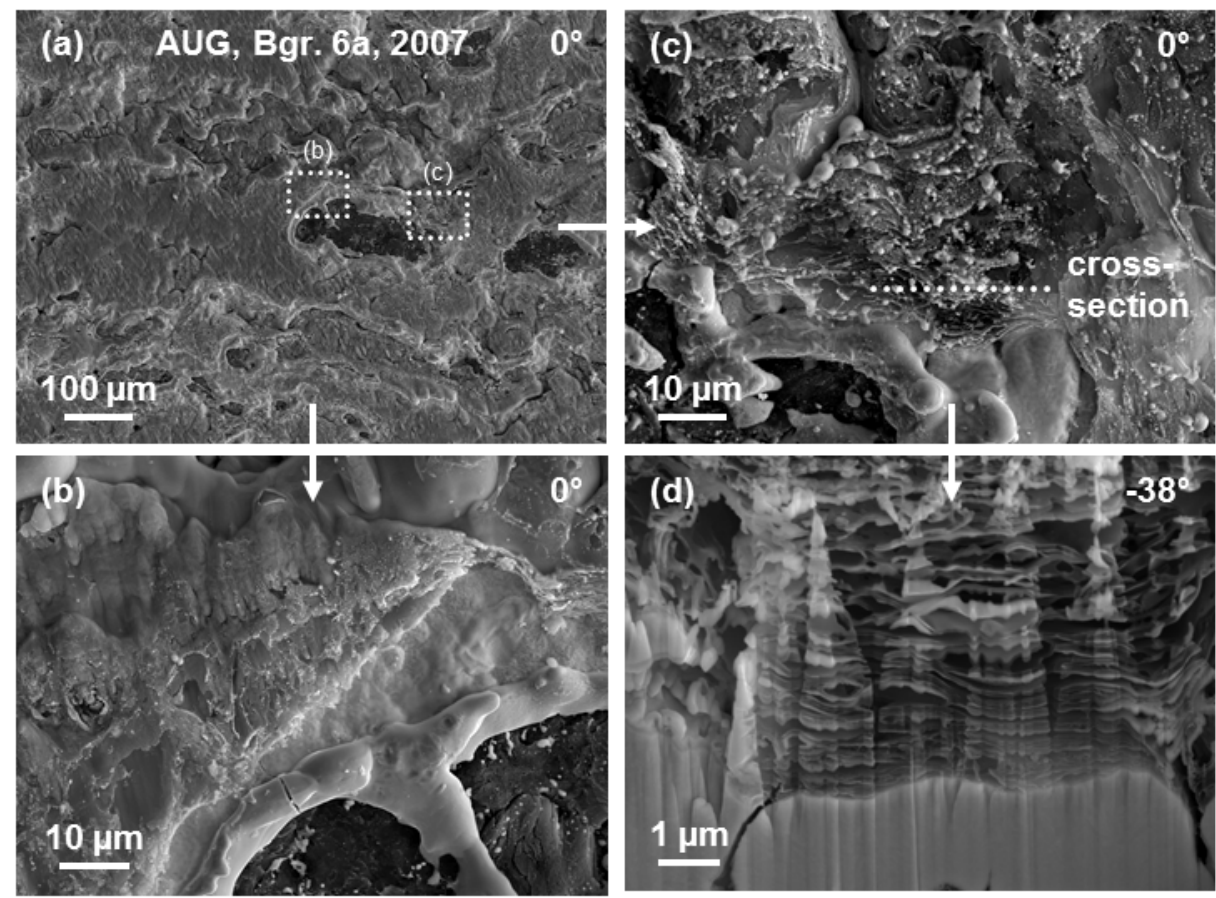

Figure 8 (two column figure): Top view SEM images of the surface of AUG divertor tiles, which suffered strong arcing (installed during campaign 2007). (a) Overview with large arc tracks. (b) and (c): Images with magnified edge of the arc track which are marked in (a). (d) Cross-section along the 


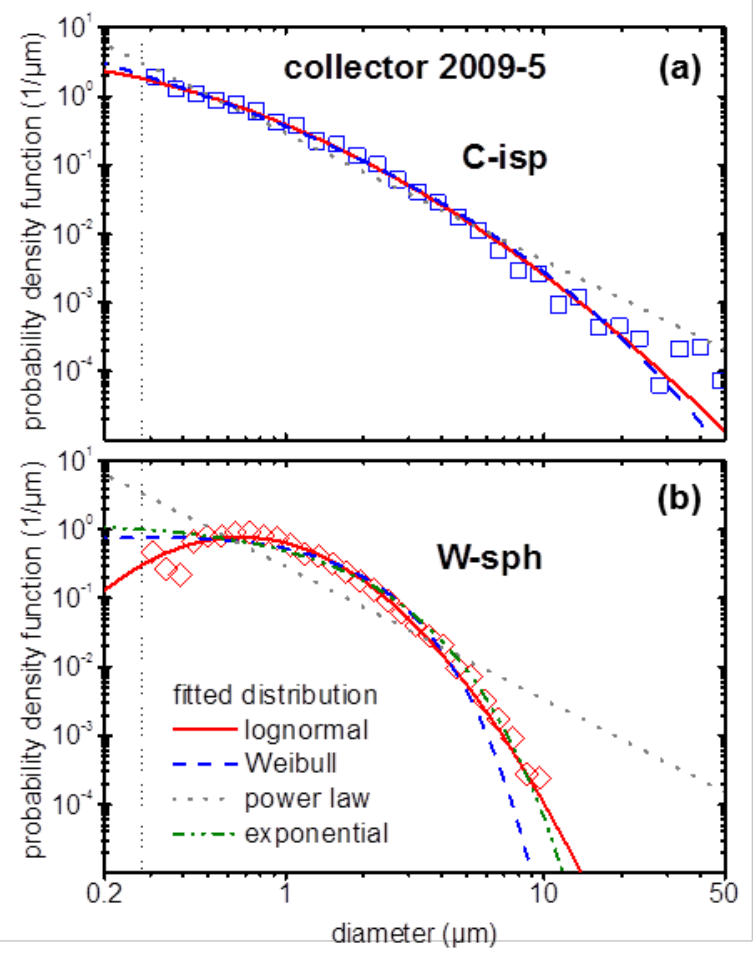

Figure 9 (one column figure): Measured PDF (log-log axes) of carbon-dominated irregularly shaped particles (a) and tungsten spheroids (b) of collector no. 5 of campaign 2009 mounted on the low field side together with the fits by lognormal, power law and Weibull distribution and for the W-sph also by the exponential distribution. Even if the data of C-isp has the appearance of roughly power law behaviour, it is better fitted by the tail of large particles of a lognormal distribution. The symbols of the measured PDF are plotted in the mid of the bins; each particle ensemble is divided into 30 bins with exponentially increasing width.

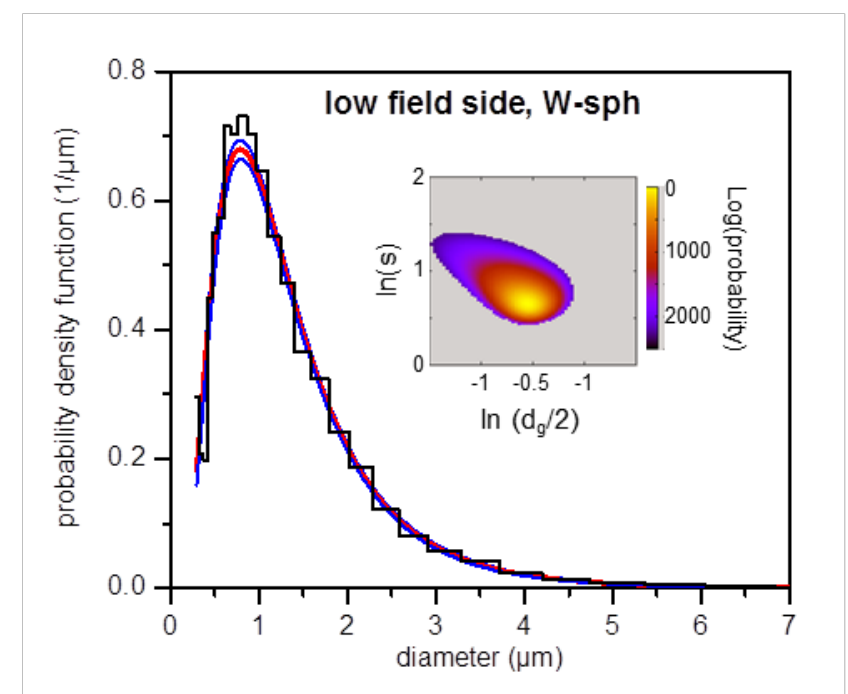

Figure 10 (one column figure): The PDF for the medium-sized ensemble of W-sph on the low field side together with its $90 \%$ confidence interval obtained from 1000 randomly taken pairs of $d_{g}$ and $s$, weighted by their likelihood (linear-linear scale). The insert is a contour plot of the logarithm of the likelihood in the space of $d_{g}$ and $s$. 


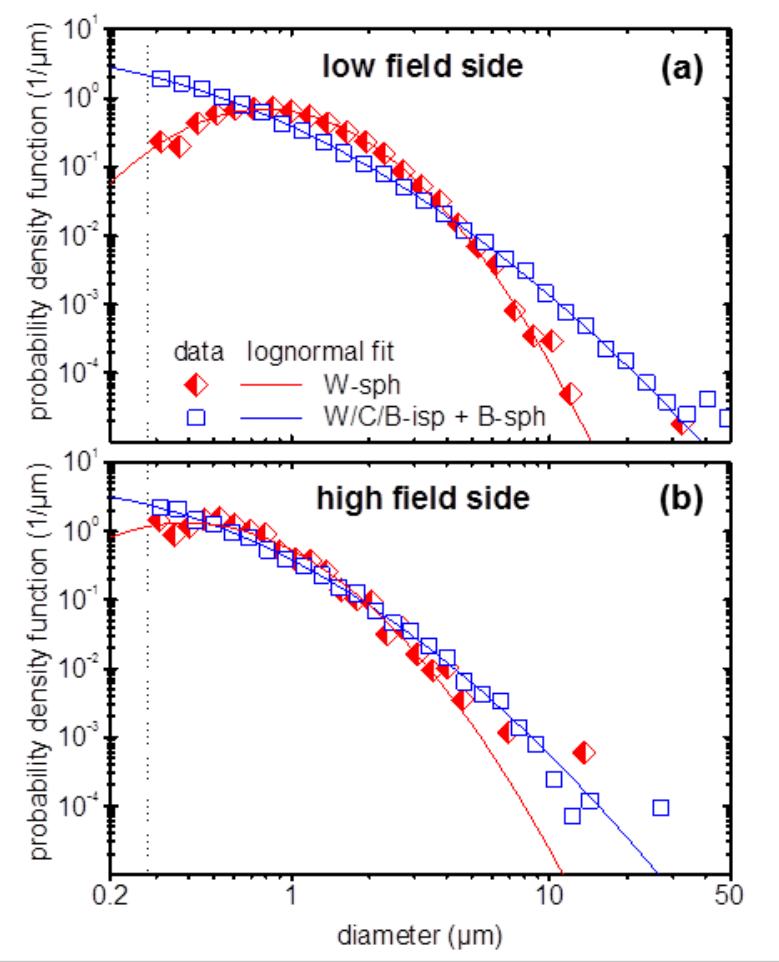

Figure 11 (one column figure): Measured and lognormal fitted PDF of tungsten spheroids and the three classes of irregularly shaped particles plus the class of boron-dominated spheroids (WCB-isp) for (a) the low and (b) the high field side (log-log plot). The symbols of the measured PDF are plotted in the mid of the bins; each particle ensemble is divided into 30 bins with exponentially increasing width. 


\section{Tables}

Table 1: Collector name, position and some general information of the collectors of the five consecutive campaigns 2007-2011. The collector name is composed of campaign year, a number, an abbreviation for the position and the sector number. The thickness of the deposit layer is measured in some cases on cross-sections prepared by FIB. If the thickness is gained by colorimetry, the value is marked with an asterisk. Note that collectors in sector 15 are not taken into account for the detailed analysis (see section 5.1) and the collector no. 2 of campaign 2008a was ruined by dropping it on the surface and, therefore, it is not analysed here.

\begin{tabular}{|c|c|c|c|c|c|c|}
\hline & Position of collector & & $\begin{array}{c}\text { Number } \\
\text { of }\end{array}$ & $\begin{array}{c}\text { Analysed } \\
\text { area }\end{array}$ & $\begin{array}{l}\text { Fraction of } \\
\text { analysed } \\
\text { area } \\
\text { covered by }\end{array}$ & $\begin{array}{l}\text { Thickness } \\
\text { of deposit }\end{array}$ \\
\hline Collector ID name & & Sector & particles & $\left(\mathrm{mm}^{2}\right)$ & particles & $(\mathrm{nm})$ \\
\hline 2007-1-PSLL11a & lower PSL & 11 & 12065 & 6.3 & $0.31 \%$ & 30 \\
\hline 2007-2-HS11 & below heat shield & 11 & 9195 & 6.5 & $0.21 \%$ & $10-30$ * \\
\hline 2007-3-APRT11 & A-port & 11 & 9539 & 14.0 & $0.16 \%$ & $10-30 *$ \\
\hline 2008a-1-PSLL11a & lower PSL & 11 & 12740 & 11.3 & $0.26 \%$ & $\sim 50 *$ \\
\hline 2008a-2-HS11 & below heat shield & 11 & - & - & - & $10-30 *$ \\
\hline 2008a-3-APRT11 & A-port & 11 & 7676 & 12.1 & $0.15 \%$ & $>100 *$ \\
\hline 2008b-1-PSLL11b & lower PSL & 11 & 8252 & 11.3 & $0.38 \%$ & $10-30 *$ \\
\hline 2008b-2-HS11 & below heat shield & 11 & 7007 & 16.5 & $0.15 \%$ & $10-30 *$ \\
\hline 2008b-3-PSLL03 & lower PSL & 3 & 12832 & 13.0 & $0.28 \%$ & $\sim 50 *$ \\
\hline 2008b-4-PSLL15 & $\begin{array}{l}\text { lower PSL, below } \\
\text { glow anode }\end{array}$ & 15 & - & 8.2 & - & >>100 * \\
\hline 2008b-5-PSLL05 & $\begin{array}{l}\text { lower PSL, below } \\
\text { ECRH }\end{array}$ & 5 & 7900 & 12.8 & $0.27 \%$ & $\sim 50 *$ \\
\hline 2009-1-HS11 & below heat shield & 11 & 9114 & 7.5 & $0.26 \%$ & $10-30$ \\
\hline 2009-2-PSLL15 & $\begin{array}{l}\text { lower PSL, below } \\
\text { glow anode }\end{array}$ & 15 & - & 4.8 & - & $>>100$ \\
\hline 2009-3-PSLL11b & lower PSL & 11 & 9883 & 11.3 & $0.23 \%$ & $10-30$ \\
\hline 2009-4-PSLL03 & lower PSL & 3 & 15486 & 15.1 & $0.40 \%$ & 70 \\
\hline 2009-5-PSLL05 & $\begin{array}{l}\text { lower PSL, below } \\
\text { ECRH }\end{array}$ & 5 & 13467 & 14.9 & $0.29 \%$ & $10-30$ \\
\hline 2011-1-DIVL11 & $\begin{array}{l}\text { below lower } \\
\text { Divertor }\end{array}$ & 11 & 11134 & 8.9 & $0.60 \%$ & $10-30 *$ \\
\hline 2011-2-PSLL11b & lower PSL & 11 & 13089 & 9.4 & $0.40 \%$ & $\sim 100 *$ \\
\hline 2011-3-PSLU11 & upper PSL & 11 & 4950 & 17.3 & $0.07 \%$ & $10-30 *$ \\
\hline 2011-4-PSLL03 & lower PSL & 3 & 19240 & 19.5 & $0.34 \%$ & $>>100 *$ \\
\hline 2011-5-HS11 & below heat shield & 11 & 11456 & 13.5 & $0.12 \%$ & $10-30 *$ \\
\hline
\end{tabular}


Table 2: Ensemble name, number of particles, fraction of analysed area which is covered by particles, fit parameters and KS statistics (fit quality) for the lognormal, power law, and Weibull distributions for all 8 classes of the high and low field side as well as some meaningful combinations for the collectors of campaign 2009. Populations that were too small to allow meaningful statistical analysis are indicated with an " $\mathrm{x}$ ". ( $d_{g}=$ geo. mean, $s=$ standard deviation $)$.

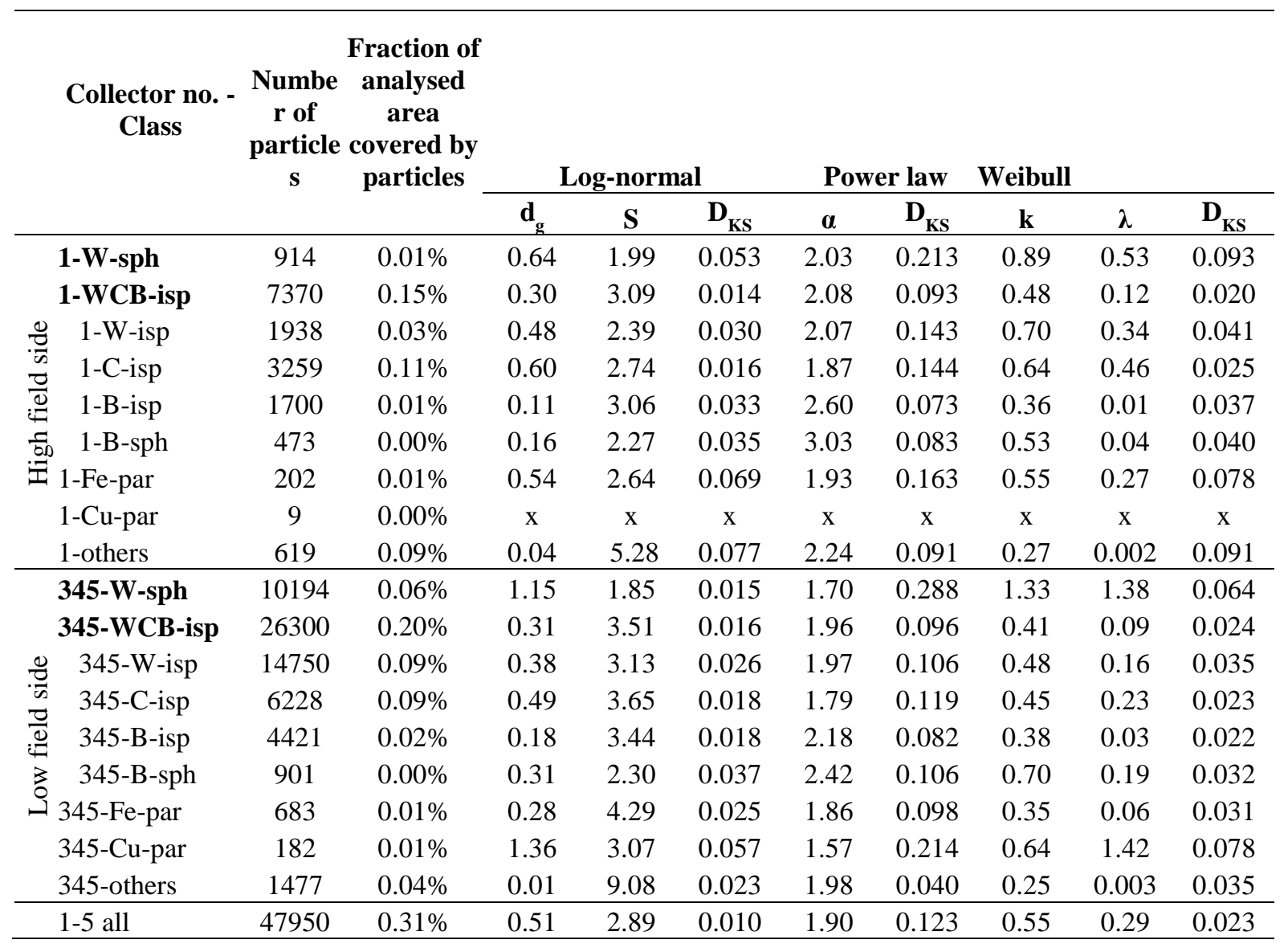


Table 3: The largest diameter ECD in each ensemble, the areal density of total volume, the surface area $(\eta=100)$, and the dust mass of the W-sph and the WCB-isp for the high (\#1) and low field side (\#345) obtained directly from the individual particles are given. From the extrapolation of the lognormal fit, the particle size $d_{75 \%}\left(d_{90 \%}\right)$, for which less than $75 \%(90 \%)$ of the volume of the ensemble is contained in particles up to the size $d_{75 \%}\left(d_{90 \%}\right)$ are calculated. In addition, the fraction $R_{d}$ of particles with ECD below $d_{\min }=0.28 \mu \mathrm{m}$ and the fraction $R_{v o l}$ of the total volume for particles with ECD below $d_{\min }$ are given. Furthermore, the total mass for the four ensembles are obtained assuming a density of $19.3 \mathrm{~g} / \mathrm{cm}^{3}$ and $3 \mathrm{~g} / \mathrm{cm}^{3}$ for the W sphere and the others, respectively. Finally, the estimate for the total mass arrived at the horizontal area represented by the Si collectors on the high field side $\left(0.7 \mathrm{~m}^{2}\right)$ and the low field side $\left(4.1 \mathrm{~m}^{2}\right)$ in AUG is gained.

\begin{tabular}{|c|c|c|c|c|c|c|c|c|c|}
\hline & \multicolumn{4}{|c|}{ directly from data } & \multicolumn{5}{|c|}{ extrapolated from lognormal fit } \\
\hline & $\begin{array}{c}\text { ECD of } \\
\text { largest } \\
\text { particle } \\
(\mu \mathrm{m})\end{array}$ & $\begin{array}{c}\text { dust } \\
\text { volume } \\
(100 \\
\left.\mu \mathrm{m}^{3} / \mathrm{m}^{2}\right) \\
\end{array}$ & $\begin{array}{c}\text { surface } \\
\text { area } \\
(100 \\
\left.\mu^{2} / \mathrm{m}^{2}\right) \\
\end{array}$ & $\begin{array}{c}\text { dust } \\
\text { mass } \\
\left(\mathrm{mg} / \mathrm{m}^{2}\right)\end{array}$ & $\begin{array}{c}d_{75 \% \mathrm{vol}} \\
\left(d_{90 \% \mathrm{vol}}\right), \\
\text { diameter } \\
\text { describing } \\
75 \% \\
(90 \%) \text { of } \\
\text { the total } \\
\text { dust } \\
\text { volume } \\
(\mu \mathrm{m}] \\
\end{array}$ & $\begin{array}{c}R_{d}, \\
\text { fraction } \\
\text { of } \\
\text { particles } \\
\text { with } \\
\text { diameter } \\
\text { below } \\
d_{\text {min }} \\
\end{array}$ & $\begin{array}{c}R_{v o l}, \\
\text { fraction } \\
\text { of } \\
\text { particle } \\
\text { volume } \\
\text { with } \\
\text { diameter } \\
\text { below } \\
d_{\text {min }} \\
\end{array}$ & $\begin{array}{c}\text { dust } \\
\text { mass } \\
\left(\mathrm{mg} / \mathrm{m}^{2}\right)\end{array}$ & $\begin{array}{c}\text { Estimate } \\
\text { d total } \\
\text { dust } \\
\text { mass } \\
\text { (mg) }\end{array}$ \\
\hline 1-W-sph & 15 & 3.8 & 5.5 & 7.4 & $4(7)$ & $11 \%$ & $0.056 \%$ & 8 & 6 \\
\hline 345-W-sph & 35 & 11.5 & 22.6 & 22.2 & $5(8)$ & $1 \%$ & $0.002 \%$ & 29 & 120 \\
\hline 1-WCB-isp & 29 & 24.6 & 43.5 & 7.4 & $29(58)$ & $47 \%$ & $0.028 \%$ & 15 & 10 \\
\hline 345-WCB-isp & 53 & 70.7 & 55.9 & 21.2 & $80(171)$ & $46 \%$ & $0.006 \%$ & 37 & 150 \\
\hline
\end{tabular}




\section{References}

[1] McCarthy KA, Petti DA, Carmack WJ and Smolik GR 1998 The safety implications of tokamak dust size and surface area Fusion Eng. Des. 42 45-52

[2] Sharpe JP, Petti DA and Bartels H-W 2002 A review of dust in fusion devices: Implications for safety and operational performance Fusion Eng. Des. 63-64 153-63

[3] Krasheninnikov SI et al 2008 Recent progress in understanding the behavior of dust in fusion devices Plasma Phys. Control. Fusion 50124054

[4] Girard JP, Gulden W, Kolbasov B, Louzeiro-Malaquias A-J, Petti D and Rodriguez-Rodrigo L 2008 Summary of the 8th IAEA Technical Meeting on Fusion Power Plant Safety Nucl. Fusion 48015008

[5] Denkevits A 2010 Hydrogen/dust explosion hazard in ITER: Effect of nitrogen dilution on explosion behavior of hydrogen/tungsten dust/air mixtures Fusion Eng. Des. 85 1059-1063

[6] Roth J et al 2009 Recent analysis of key plasma wall interactions issues for ITER J. Nucl. Mater. 390-391 1-9

[7] Winter J 1998 Dust in fusion devices-experimental evidence, possible sources and consequences Plasma Phys. Control. Fusion 40 1201-10

[8] Federici G, Skinner CH, Brooks JN, Coad JP, Grisolia C, Haasz AA, Hassanein A, Philipps V, Pitcher CS, Roth J, Wampler WR and Whyte DG 2001 Plasma-material interactions in current tokamaks and their implications for next step fusion reactors Nucl. Fusion 411967

[9] Rudakov DL et al 2008 Dust measurements in tokamaks Review Of Scientific Instruments 79 10F303

[10] Ratynskaia S, Castaldo C, Bergsåker H and Rudakov D 2011 Diagnostics of mobile dust in scrape-off layer plasmas Plasma Phys. Control. Fusion 53074009

[11] Krasheninnikov SI, Smirnov RD and Rudakov DL 2011 Dust in magnetic fusion devices Plasma Phys. Control. Fusion 53083001

[12] Krasheninnikov SI 2011 Multifaceted physics of edge plasma in magnetic fusion devices Plasma Phys. Control. Fusion 53074017

[13] Fortov VL and Morfil GE (Eds) 2010 Complex and Dusty Plasmas: From Laboratory to Space (Boca Raton, London, New York: CRC Press Taylor \& Francis Group)

[14] Shukla PK and Mamun AA 2002 Introduction to Dusty Plasma Physics” 2002 (Bristol, Philadelphia: IoP Institute of Physics Publishing)

[15] Boufendi L and Bouchoule A 1994 Particle nucleation and growth in a low-pressure argonsilane discharge Plasma Sources Science \& Technology 3 262-7

[16] Hollenstein C 2000 The physics and chemistry of dusty plasmas Plasma Phys. Control. Fusion 42 R93-104

[17] Annaratone BM, Jacob W, Arnas C and Morfill GE 2009 Critical Review of Complex Plasma (Dusty Plasma) Diagnostics and Manipulation Techniques for the Fusion Community and Others IEEE Transactions Plasma Science 37 270-80

[18] Winter J 2004 Dust in fusion devices-a multi-faceted problem connecting high- and lowtemperature plasma physics Plasma Phys. Control. Fusion 46 B583-92

[19] Carmack WJ, McCarthy KA, Petti DA, Kellman AG and Wong CPC 1998 Collection and analysis of particulate from the DIII-D Tokamak Fusion Eng. Des. 39, 477-83

[20] Peacock AT, Andrew P, Cetier P, Coad JP, Federici G, Hurd HF, Pick MA and Wu CH 1999 Dust and flakes in the JET MkIIa divertor, analysis and results J. Nucl. Mater. 266-9 423-8 
[21] Carmack WJ, Anderl RA, Pawelko RJ, Smolik GR and McCarthy KA 2000 Characterization and analysis of dusts produced in three experimental tokamaks: TFTR, DIII-D, and Alcator CMod Fusion Eng. Des. 51-52, 477-84

[22] Rubel M, Cecconello M, Malmberg JA, Sergienko G, Biel W, Drake JR, Hedqvist A, Huber A and Philipps V 2001 Dust particles in controlled fusion devices: morphology, observations in the plasma and influence on the plasma performance Nuclear Fusion 41 1087-99

[23] Chappuis P, Tsitrone E, Mayne M, Armand X, Linke J, Bolt H, Petti D and Sharpe JP "Dust characterization and analysis in Tore-Supra” 2001 J. Nucl. Mater. 290-293 245-249

[24] Muto S, Tanabe T, Hirota A, Rubel M, Philipps V and Maruyama T "TEM and EELS characterization of carbon dust and co-deposited layers from the TEXTOR tokamak” 2002 Journal of Nuclear Materials 307-311 1289-1293

[25] Sharpe JP, Rohde V and The ASDEX-Upgrade Experiment Team, Sagara A, Suzuki H, Komori A, Motojima O and The LHD Experimental Group "Characterization of dust collected from ASDEX-Upgrade and LHD” 2003 J. Nucl. Mater. 313-316 455-9

[26] Sharpe JP, Humrickhouse PW, Skinner CH, NSTX Team, Tanabe T, Masaki K, Miya N, JT60U Team and Sagara A 2005 Characterization of dust collected from NSTX and JT-60U J. Nucl. Mater. 337-339 1000-4

[27] Smirnov RD, West WP, Krasheninnikov SI, Pigarov AY, Rosenberg M and Bray BD 2007 Laser-dust interaction and dust size distribution measurements on DIII-D Physics of Plasmas 14112507

[28] Ivanova D, Rubel M, Philipps V, Freisinger M, Huang Z, Penkalla H, Schweer B, Sergienko G, Sundelin P and Wessel E 2009 Survey of dust formed in the TEXTOR tokamak: structure and fuel retention Phys. Scr. T138 014025

[29] Koga K, Iwashita S, Kiridoshi S, Shiratani M, Ashikawa N, Nishimura K, Sagara A and Komori A 2009 Characterization of Dust Particles Ranging in Size from $1 \mathrm{~nm}$ to $10 \mu \mathrm{m}$ Collected in the LHD Plasma and Fusion Research 4034

[30] Roubin P, Pégourié B, Smirnov R, Martin C, Richou M, Marandet Y, Pardanaud C,Brosset C, and Gunn J 2009 Analysis of carbon deposited layer growth processes in Tore Supra J. Nucl. Mater. 390-391 49-52

[31] Morfill G, Räth C, Li Y-F, Hu JS, Ling BL, X Gao X and Horányi M 2009 Dust capture experiment in HT-7 New Journal of Physics 11113041

[32] Rudakov DL et al 2009 Dust studies in DIII-D and TEXTOR Nuclear Fusion 49085022

[33] Arnas C et al 2010 Similarities and differences between dust produced in laboratory plasmas and in the MAST and Tore Supra tokamaks Plasma Phys. Control. Fusion 52124007

[34] Asakura N, Hayashi T, Ashikawa N, Hatae T and Nakano T 2011 Measurements of carbon dust property in experiment and post-campaign sampling on JT-60U tokamak Fusion Science And Technology 60 1572-1575

[35] Litnovsky A et al 2013 Dust investigations in TEXTOR Impact of dust on plasma-wall interactions and on plasma performance J. Nucl. Mater. 438 S126-132

[36] Smirnov RD, Krasheninnikov SI, Yu JH, Pigarov AY, Rosenberg M and Terry JL 2009 On visibility of carbon dust particles in fusion plasmas with fast framing cameras Plasma Phys. Control. Fusion 51055017

[37] Smirnov RD, Krasheninnikov SI, Pigarov AY, Roquemore AL, Mansfield DK and Nichols J 2011 Modeling of dust impact on tokamak edge plasmas J. Nucl. Mater. 415 S1067-72

[38] Lazzaro E, Proverbio I, Nespoli F, Ratynskaia S, Castaldo C, deAngelis U, DeAngeli M, Banon J-P and Vignitchouk L 2012 Transport and effects of ferromagnetic dust in a tokamak with a metallic vessel Plasma Phys. Control. Fusion 54124043 
[39] Tomita Y, Pigarov AY, Smirnov RD, Krasheninnikov SI, Ohno N and Uesugi Y 2009 Release conditions of dust particle from plasma-facing wall in oblique magnetic field $\mathrm{J}$. Nucl. Mater 390-391 164-7

[40] Smirnov RD, Krasheninnikov SI, Pigarov AY, Benson DJ, Rosenberg M and Mendis DA 2009 Modeling of velocity distributions of dust in tokamak edge plasmas J. Nucl. Mater. 390$39184-7$

[41] Liu J, Chen L, Mao A, Sun G and Duan P 2013 Charging, movement and lifetime characteristics of dust in magnetic fusion devices Vacuum 88 177-81

[42] Jovanovic D, Fiore G and de Angelis U 2013 A self-consistent picture for hyper-velocity metal dust in FTU Nuclear Fusion 53033008

[43] Neu R et al 2009 Ten years of $\mathrm{W}$ programme in ASDEX Upgrade-challenges and conclusions Phys. Scr. T138 014038

[44] Neu R et al 2007 Operational conditions in a W-clad tokamak” J. Nucl. Mater. 367-370 1497-502

[45] Rohde V 2008 Demonstration of Diagnostic Techniques for time resolved Dust Measurement in Tokamaks TW6-TPP-DUSMEAS EFDA Final Report

[46] Balden M, Rohde V and the ASDEX Upgrade Team 2008 First investigation of the inner morphology of dust particles from ASDEX Upgrade”, Post-deadline poster at $18^{\text {th }}$ Int. Conf. on Plasma Surface Interactions (2008), Toledo, Spain http://psi2008.ciemat.es/documents/Book_of_abstracts3.pdf

[47] Rohde V, Balden M, Lunt T and ASDEX Upgrade Team 2009 Dust investigations at ASDEX Upgrade Phys. Scr. T136 014024

[48] Balden M, Endstrasser N, Rohde V, Rasinski M, Lindig L, Neu R and ASDEX Upgrade Team 2010 Dust Collection Strategy and Inner Morphology of Dust Particles in ASDEX Upgrade EXD P3-03, IAEA FEC, Daejon http://www-pub.iaea.org/mtcd/meetings/PDFplus/2010/cn180/cn180_BookOfAbstracts.pdf

[49] Endstrasser N, Brochard F, Rohde V, Balden M, Lunt T, Bardin S, Briançon JL, Neu R and the ASDEX Upgrade Team 2011 Video tracking and post-mortem analysis of dust particles from all tungsten ASDEX-Upgrade J. Nucl. Mater. 415 S1085-8

[50] Endstrasser N, Rohde V, Balden M, Humrickhouse P, Toussaint Uv, Braams B, Chung, H Neu $\mathrm{R}$ and the ASDEX Upgrade Team 2011 Comparative study of the dust particle population sampled during four consecutive campaigns in all-tungsten ASDEX Upgrade Phys. Scr. T145 014021

[51] Fortuna-Zalesna E, Grzonka J, Rasinski M, Balden M, Rohde V, Kurzydlowski KJ, ASDEX Upgrade Team 2013/4 Characterization of dust collected after plasma operation of all tungsten ASDEX Upgrade Phys. Scr. T159 014066

[52] Merkus HG 2009 Particle Size Measurements: Fundamentals, Practice, Quality” (New York: Springer2009) Chapter 1-7

[53] Humrickhouse PW and Sharpe JP 2008 Dust mobilization and transport for loss of vacuum accidents Fusion Eng. Des. 83 1721-4

[54] Klinkov SV, Kosarev VF and Rein M 2005 Cold spray deposition: Significance of particle impact phenomena Aerospace Sci. Technol. 9 582-91

[55] Almeida-Prieto S, Blanco-Mendez J and Otero-Espinar J 2007 Microscopic image analysis techniques for the morphological Euro. J. Pharm. Biopharm. 67 766-76

[56] Hentschel ML and Page NW 2003 Selection of descriptors for particle shape characterization Part. Part. Syst. Charact. 20 25-38 
[57] ImageJ (Image Processing and Analysis in Java): http://rsbweb.nih.gov/ij/

[58] IAEA data base: http://www-amdis.iaea.org/CRP/Dust/

[59] Rohde V, Dux R, Kallenbach A, Krieger K, Neu N and ASDEX Upgrade Team 2007 Wall conditioning in ASDEX Upgrade J. Nucl. Mater. 363-5 1369-74

[60] Stenzel O 1996 Das Dünnschichtspektrum (Berlin: Akademie) p 63

[61] Balden M, Rohde V, Lindig S, Manhard A, Krieger K and ASDEX Upgrade Team 2013 Blistering and re-deposition on tungsten exposed to ASDEX Upgrade divertor plasma J. Nucl. Mater. 438 S220-3

[62] Samsonov D and Goree J 1999 Particle growth in a sputtering discharge J. Vac, Sci. Technol. A 17 2835-40

[63] Urban FK, Hosseini-Tehrani A, Griffiths P, Khabari A, Kim YW and Petrov I 2002 Nanophase films deposited from a high-rate, nanoparticle beam J. Vac. Sci. Technol. B20 995-9

[64] Rohde V, Endstrasser N, Toussaint U v, Balden M, Lunt T, Neu R, Hakola A, Bucalossi J and ASDEX Upgrade Team 2011 Tungsten Erosion By Arcs In ASDEX Upgrade J. Nucl. Mater. 415 S46-50

[65] ASDEX Upgrade Team 2008 Private communication: Exfoliation of thick W-coating of a divertor tile end of campaign 2008a of ASDEX Upgrade

[66] Krieger K, Lunt T, Dux R, Janzer A, Kallenbach A, Müller HW, Neu R, Pütterich T, Rohde V and The ASDEX Upgrade Team 2011Induced tungsten melting events in the divertor of ASDEX Upgrade and their influence on plasma performance J. Nucl. Mater. 415 S297-300

[67] Laux M, Balden M, and Siemroth P 2013/4 Modification of arc emitted W-particles in a model scrape-off layer plasma Phys. Scr. T159 014026

[68] Thevenot F 1990 Boron Carbide-A Comprehensive Review Journal of the European Ceramic Society 6 205-25

[69] Portnoi KI, Romashov VM, Levinskii YV and Romanovich IV 1967 Phase diagram of the system tungsten-Boron Powder Metallurgy And Metal Ceramics 6 398-402 (Translated from 1967 Poroshkovaya Metallurgiya 5 75-80)

[70] Kurlov AS and Gusev AI 2006 Tungsten Carbides and W-C Phase Diagram Inorganic Materials 42 121-7

[71] Laux M, Schneider W, Jüttner B, Lindig S, Mayer M, Balden M, Beilis I and Djakov B 2005 Modification of tungsten layers by arcing J. Nucl. Mater. 337-339 1019-23

[72] Laux M, Schneider W, Jüttner B, Balden M, Lindig S, Beilis II and Djakov BE 2005 Ignition and Burning of Vacuum Arcs on Tungsten Layers IEEE Transaction on Plasma Science 33 $1470-5$

[73] Daalder JE 1976 Components of cathode erosion in vacuum arcs J. Phys. D: Appl. Phys. 9 2379-95

[74] Hinds WC 1999 Aerosol Technology (New York: Wiley-Interscience)

[75] Clauset A, Shalizi CR and Newman MEJ 2009 Power-Law Distributions in Empirical Data SIAM Review 51 661-703

[76] Humrickhouse PW, Balden, M and Rohde, V 2014 Size distribution analysis methods and their application to ADEX-Upgrade dust Fusion Engineering and Design, submitted

[77] Rosin P and Rammler E 1933 Laws governing the fineness of powdered coal Journal of the Institute of Fuel 7 29-36

[78] R Development Core Team 2010 R: A Language and Environment for Statistical Computing”, 
R Foundation for Statistical Computing, Vienna, Austria, URL http://www.R-project.org, ISBN 3-900051-07-0

[79] Sugiyama K et al 2010 Deuterium inventory in the full-tungsten divertor of ASDEX Upgrade Nucl. Fusion 50035001 